\title{
Synthesis and Implementation of Sensor-Less Active Shunt Controllers for Electromagnetically Actuated Systems
}

\author{
Andrew J. Fleming, Member, IEEE, S. O. Reza Moheimani, Senior Member, IEEE, and Sam Behrens
}

\begin{abstract}
Electromagnetic transducers have been used extensively for active feedback control of mechanical vibration. In this paper, to reduce vibration, we demonstrate a new technique where an electrical impedance is connected to the terminals of an electromagnetic transducer. By measuring the coil terminal voltage and applying a related current, or vice-versa, vibration in the mechanical host system can be controlled. The task of impedance design is cast as a standard multi-input-multi-output (MIMO) control problem to facilitate synthesis by standard methods such as linear quadratic regulator (LQR) or $\mathcal{H}_{2}$. Potential applications include: vehicle suspension systems, vibration isolation platforms, and the control of enclosed-sound fields. Active admittance or impedance controllers require no external sensors. The presented techniques are verified experimentally through the application to a single-degree-of-freedom (DOF) system.
\end{abstract}

Index Terms-Active, damping, electromagnetic, noise control, self-sensing, sensor-less, shunt, vibration.

\section{INTRODUCTION}

$\mathbf{E}$ LECTROMAGNETIC transducers [1]-[3] can be used as actuators, sensors, or both. When a current is applied to the terminals of an electromagnetic transducer, a force is exerted, conversely, when a transducer experiences a velocity, an open-circuit voltage is induced. Piezoelectric transducers [4] exhibit similar electromechanical properties but are characterized by a high-mechanical impedance. Electromagnetic transducers are capable of significantly greater strokes, typically in the millimeter range compared to the micrometer range.

Physically, electromagnetic devices are robust and can be manufactured on all scales from microelectromechanical systems (MEMS) devices, to large 100-kN electrodynamic shakers. Such transducers have found application in the fields of: car suspension systems [5], isolation platforms [6], [7], acoustic speakers [8], magnetic levitation [9], [10], and magnetic bearings [11].

In analogy to the technique of piezoelectric self-sensing [12], [13], a recent literature has also developed on the topic of electromagnetic self-sensing actuators [3], [9]-[11], [14]. This technique involves estimation of the system velocity from measurements of the transducer current and voltage. An example of this

Manuscript received March 11, 2003. Manuscript received in final form July 16, 2004. Recommended by Associate Editor A. Kelkar. This work was supported by the Australian Research Council.

The authors are with the School of Electrical Engineering and Computer Science, The University of Newcastle, Callaghan N.S.W. 2308, Australia (e-mail: andrew@ee.newcastle.edu.au).

Digital Object Identifier 10.1109/TCST.2004.839565 technique can be found in [15], where the acoustic pressure of an enclosed-sound field is estimated from the measured current flowing through an actuating speaker coil. A feedback loop, driving the speaker voltage, is constructed around the estimate to minimize the acoustic response of the enclosure.

In this paper, we demonstrate the modeling, design, and implementation of active impedance and admittance controllers for electromagnetically actuated systems. By measuring the coil terminal voltage and controlling the resultant current, or vice-versa, effectively implementing some electrical impedance or admittance, it is possible to gain control over a coupled mechanical system. After revealing the underlying feedback structure, the problem is cast as a standard multi-input-multi-output (MIMO) control problem. The application of synthesis techniques such as linear quadratic regulator (LQR) and $\mathcal{H}_{2}$ is straightforward. Using this technique, the need for external sensors is eliminated, significantly reducing the cost, complexity, and sensitivity to transducer failure that in many applications, may preclude the use of an active control system.

Although, the focus is on control of a system similar to an isolation column, the generality of the modeling and design framework is intended to be extensible to a large class of mechanical systems. Such applications include: MIMO vehicle suspension systems [5], vibration isolation platforms [6], [7], inertial vibration controllers [16], and the control of enclosed-sound fields. Active admittance or impedance controllers require no external sensors, are capable of minimizing a prespecified performance objective, and can also be used to estimate physical variables dynamically related the system states such as velocity.

Experimentally, the presented techniques are verified through their application to a single-degree-of-freedom (DOF) mechanical system.

This paper is presented in five sections. In Section II, we begin with the modeling of mechanical, electromagnetic, and composite systems. We then present a method in Section III for the design of active impedance and admittance controllers to minimize a time domain $(\mathrm{LQR})$ and frequency domain $\left(\mathcal{H}_{2}\right)$ performance objective. The presented techniques are then applied to an experimental electromagnetic system in Section IV. Finally, the paper is concluded in Section V.

\section{ModELING}

This section introduces a modeling technique for the design and analysis of shunted electromechanical systems. Although 


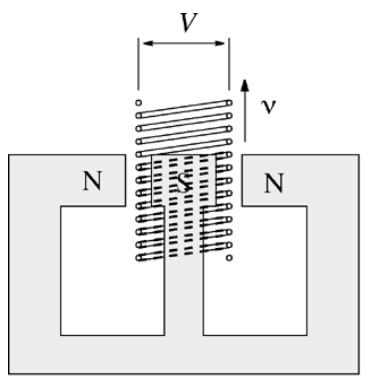

(a)

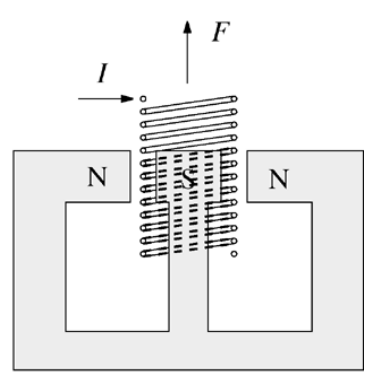

(b)
Fig. 1. Electromagnetic transducer. (a) Sensing. (b) Actuating.

the focus is on a single DOF system, the process is quite general and can easily be extended to more complex mechanical systems.

\section{A. Electromagnetic System}

When an electrical conductor moves in a magnetic field as shown in Fig. 1(a), a voltage $V$ proportional to the velocity $\nu$ is induced and appears across the terminals of the coil

$$
V \propto \nu
$$

Specifically

$$
\frac{V}{\nu}=D l
$$

where $D$ is the magnetic flux (in Teslas), $l$ is the length of the conductor (in meters), and $\nu$ is the velocity of the conductor relative to the magnetic field (in $\mathrm{ms}^{-1}$ ). A permanent magnet is usually the source of the magnetic field. In another configuration, the coil is kept stationary and the magnet is made to move.

Assuming the coil is exposed to a field of constant flux density and the relative displacement is small, (2) can be rewritten [2] as

$$
\frac{V}{\nu}=\frac{F}{I}=D l=C_{n}
$$

where $F$ denotes the force (in Newtons) acting on the coil carrying a current $I$ (in amps), and $C_{n}$ is the ideal electromechanical coupling coefficient.

As shown in Fig. 1(b), when the coil is employed as a force actuator, (3) relates the induced force to an applied current. Electrodynamic shakers and acoustic speakers operate on this principle.

As shown in Fig. 2, the coil can be modeled as the series connection of an inductor $L$, a resistor $R$, and a dependent voltage source $V_{e}$ [3]. When coupled to a mechanical system, the induced electromotive-force (emf) and, hence, mechanical velocity can be determined from the open-circuit coil terminal voltage.

\section{B. Mechanical System}

The general model of a mechanical system is shown in Fig. 3. In addition to various application specific inputs and outputs, to couple an electromagnetic transducer, the model requires a force

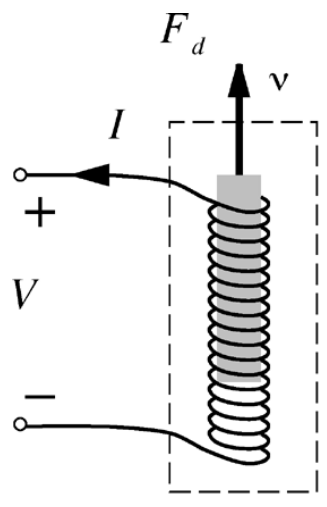

(a)

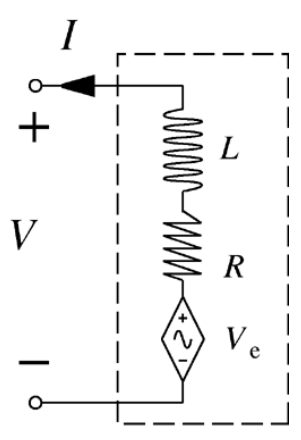

(b)
Fig. 2. (a) Mechanical and (b) electrically equivalent model of an electromagnetic transducer.

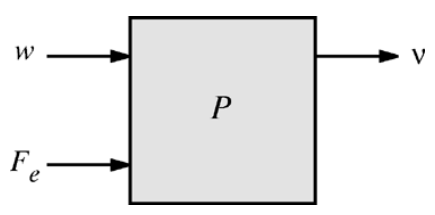

Fig. 3. General mechanical plant model. (a)

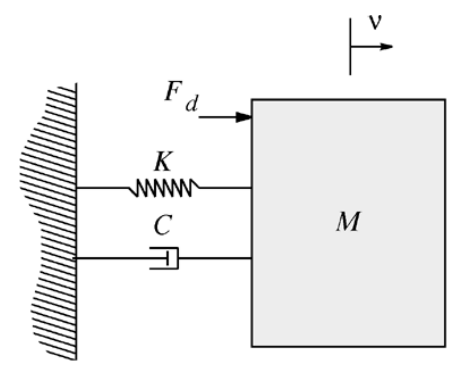

(b)

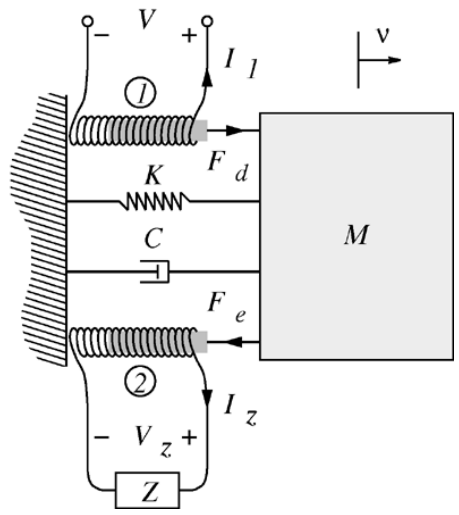

Fig. 4.(a) Mass-spring-damper system. (b) Coupled to two electromagnetic coils.

input $F_{e}$ and a velocity output $\nu$. In a typical scenario, the model would also describe the influence of a specific disturbance input $w$.

In many cases where vibration becomes an issue, the mechanical structure can be modeled as a simple mass-spring-damper system as shown in Fig. 4(a). Examples include, but are not limited to: isolation columns, magnetic bearings, and suspension systems. 


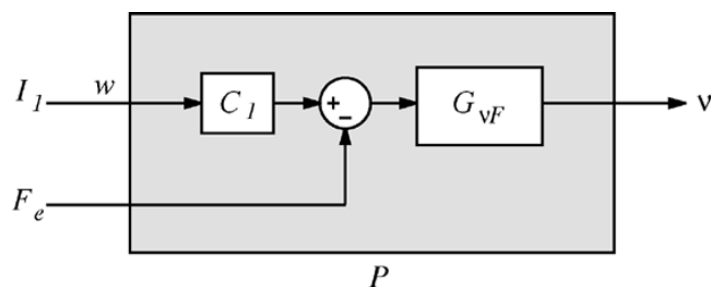

Fig. 5. Mechanical plant $P$ with a current disturbance $I_{1}$, and command input $F_{e}$.

The equation of motion for a forced 1-DOF system is

$$
M \dot{\nu}(t)+C \nu(t)+K d(t)=F_{d}(t)
$$

where $F_{d}(t)$ is the applied force, $M$ is the equivalent mass (in $\mathrm{kg}$ ), $K$ is the spring constant (in $\mathrm{N} / \mathrm{m}$ ), $C$ is the damping constant (in $\mathrm{Ns} / \mathrm{m}$ ), and $\dot{\nu}(t), \nu(t)$, and $d(t)$ are the acceleration, velocity, and displacement, respectively. The dimensionless representation of (4) is

$$
\dot{\nu}(t)+2 \zeta_{n} \omega_{n} \nu(t)+\omega_{n}^{2} d(t)=f_{d}(t)
$$

where $\omega_{n}$ is the natural frequency of the system and $\zeta_{n}$ is the damping ratio. Note that $\omega_{n}=\sqrt{K / M}, \zeta_{n}=C / \sqrt{4 M K}$ and $f_{d}(t)=F_{d}(t) / M$. In the Laplace domain, the transfer function $G_{\nu F}(s)$ from an applied force to the resulting velocity is

$$
G_{\nu F}(s)=\frac{\nu(s)}{F_{d}(s)}=\frac{s \frac{1}{M}}{s^{2}+s \frac{C}{M}+\frac{K}{M}} .
$$

In later sections, the following minimal state-space model for $G_{\nu F}(s)$ will also be required:

$$
\begin{aligned}
\dot{x}_{p}(t) & =\mathbf{A}_{p} x_{p}(t)+\mathbf{B}_{p} F_{e}(t) \\
\nu(t) & =\mathbf{C}_{p} x_{p}(t) .
\end{aligned}
$$

Consider Fig. 4(b), where a single DOF system is coupled to two electromagnetic coils. Coil 1 is used to introduce a force disturbance, and coil 2, to control the resulting vibration. The corresponding mechanical plant model $P$ is shown in Fig. 5. The general constants $C_{1}$ through $C_{4}$ represent the various electromechanical coupling constants as defined in (8). The constants are defined individually as the two coils will neither be perfectly matched nor have exactly identical force-current or velocity-voltage ratios. By definition

$$
\begin{aligned}
C_{1}=\frac{F_{d}}{I_{1}} & C_{2}=\frac{V_{e_{1}}}{\nu} \\
C_{3}=\frac{F_{e}}{I_{2}} & C_{4}=\frac{V_{e_{2}}}{\nu} .
\end{aligned}
$$

Using the constants defined in (8), the electromagnetic system $E$ associated with coil 2 is shown in Fig. 6(a) and (b) for both combinations of voltage and current drive, respectively.

\section{Shunted Composite Electromechanical System}

A mechanical system $G_{\nu F}(s)$ coupled to a shunted electromagnetic transducer as shown in Fig. 4(b) is now considered. In (a)

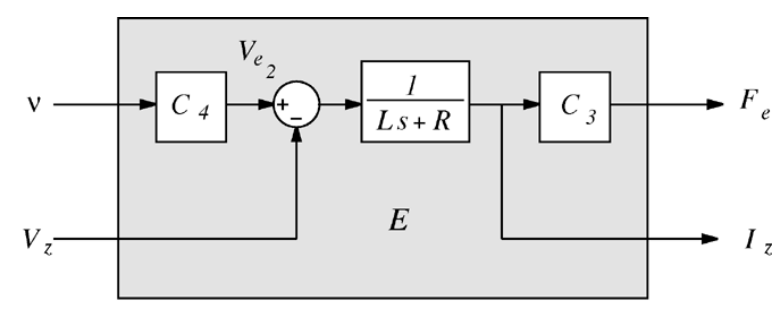

(b)

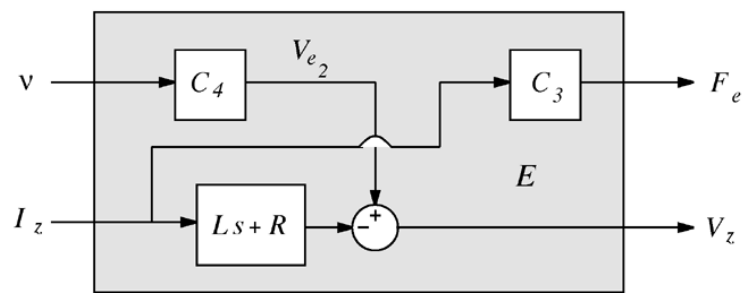

Fig. 6. Block diagram representation of a (a) voltage driven and (b) current driven electromagnetic actuator.

this case, where coil 1 is used to introduce a force disturbance $F_{d}$, coil 2, the shunted coil, is used to reduce the resulting vibration.

Within the modeling framework introduced in the previous two subsections, i.e., by treating the mechanical plant and shunted electromagnetic coil as shown in Figs. 5 and 6, it is a simple and intuitive task to construct the composite system. For both the current and voltage driven coil models, the interconnection of each electromagnetic system with the mechanical plant model is shown in Figs. 7(a) and 8(a).

In Fig. 7(a) the admittance $Y(s)$, interpreted simply as the transfer function relating the coil terminal voltage to current, appears like a feedback controller for the electromechanical system. By concatenating the mechanical and electromagnetic systems, $P$ and $E$, as shown in Fig. 7(b), the composite system is cast as a typical regulation problem for the abstracted system $G$. It is easily shown that the closed-loop transfer function from an applied disturbance current $I_{1}(s)$ to the resulting plunger velocity $\nu$ is

$$
\frac{\nu(s)}{I_{1}(s)}=\frac{G_{\nu F}(s) C_{1}}{1+K(s) C_{3} C_{4} G_{\nu F}(s)}
$$

where $K(s)$, the equivalent velocity feedback controller is

$$
K(s)=\frac{Y(s)}{1+Y(s)(L s+R)} .
$$

Similarly, in Fig. 8(a) the impedance $Z(s)$, interpreted simply as a transfer function relating the coil terminal current to voltage, appears like a feedback controller for the electromechanical system. By concatenating the mechanical and electromagnetic systems, $P$ and $E$, as shown in Fig. 8(b), the composite system is cast as a typical regulation problem for the abstracted system $G$. In this case, the equivalent velocity feedback controller is

$$
K(s)=\frac{1}{L s+R+Z(s)} .
$$


(a)

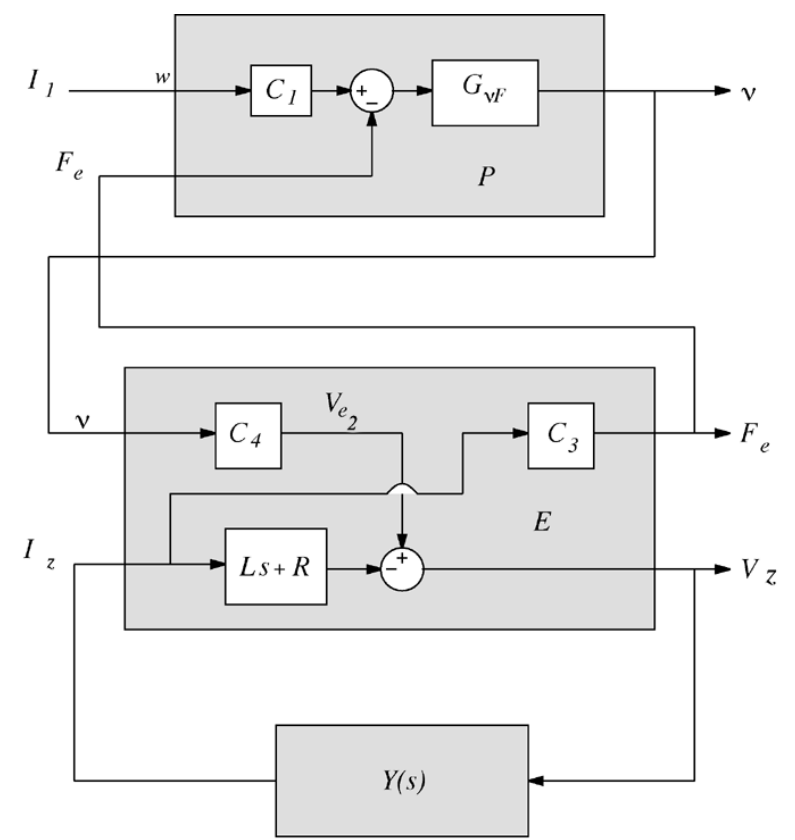

(b)

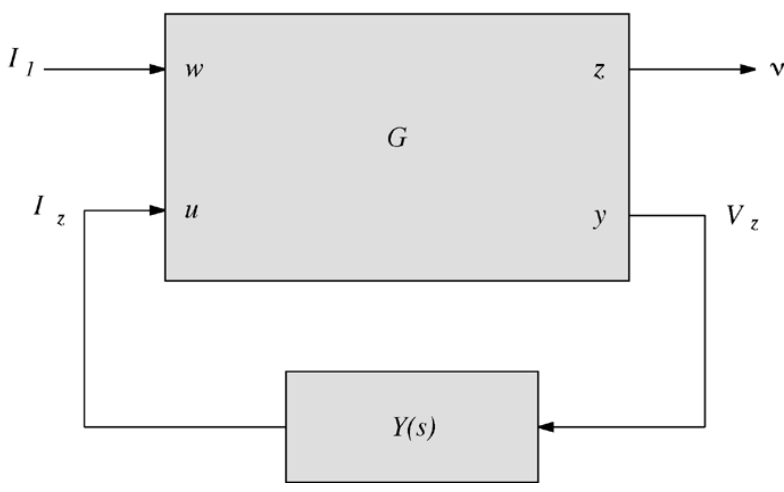

Fig. 7. (a) Shunt admittance controlled electromechanical system. (b) In generalized plant/controller form.

\section{CONTROL DESIGN}

As shown in Figs. 7 and 8, and in (9), an impedance or admittance can be viewed as parameterizing an equivalent velocity feedback controller for the mechanical system $G_{\nu F}(s)$. The following subsections introduce a number of techniques for the synthesis of active impedance and admittance controllers designed to minimize structural vibration.

\section{A. Passive Shunt Circuits}

Alike the piezoelectric analogy, a resonant shunt circuit can be used to compensate for the inductive source impedance over a small frequency band. In [17], the circuit shown in Fig. 9 was shown to significantly attenuate a lightly damped mechanical system. The circuit requires a negative resistance to cancel the natural resistance of the coil. Similar impedance controllers can be found in [18].

Resonant shunt circuits provide a fixed performance objective, they introduce additional dynamics that attenuate a highly resonant structural mode. Consider three typical electromagnetic applications: isolation support [6], [7], inertial vibration control [16], and acoustic noise control. These systems are likely to contain a naturally high degree of damping, resonant (a)

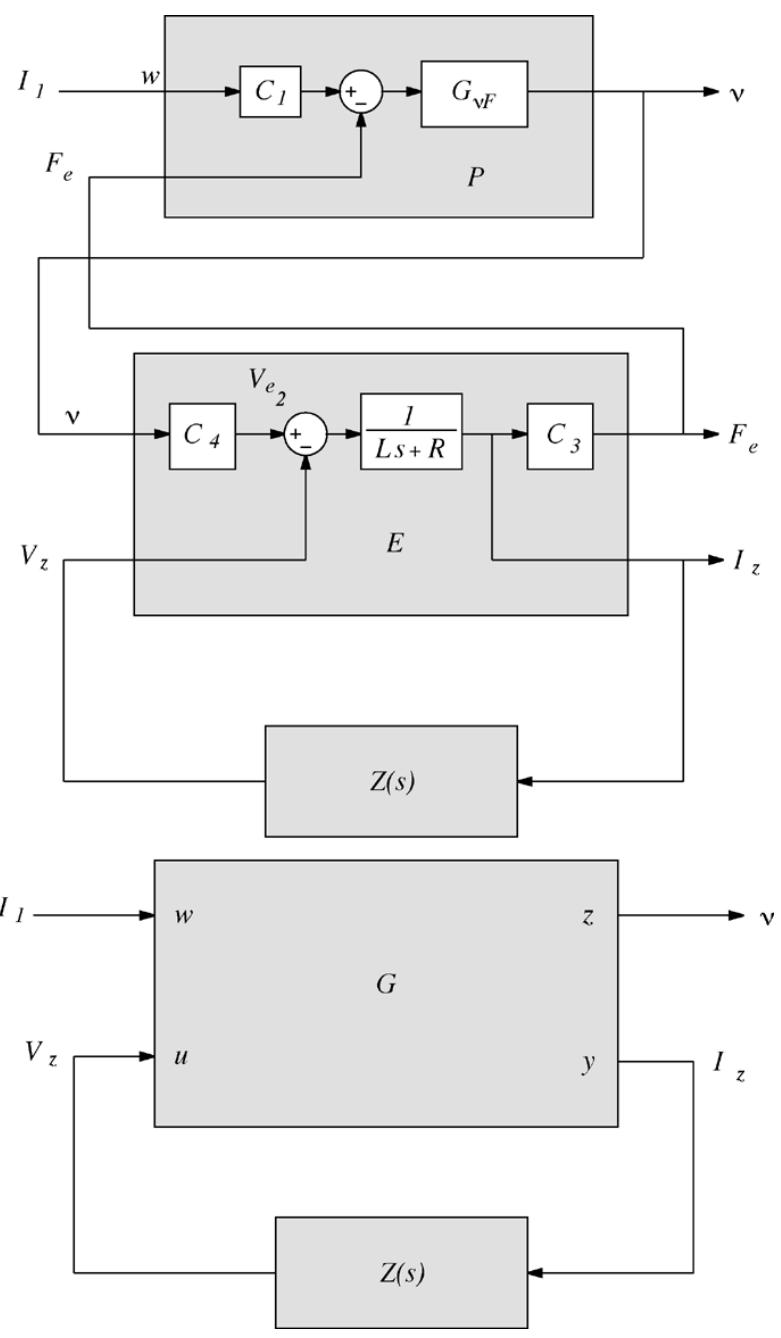

Fig. 8. (a) Shunt impedance controlled electromechanical system. (b) In generalized plant/controller form.

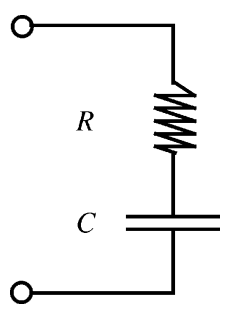

Fig. 9. Resonant shunt circuit for electromagnetic transducers.

shunt circuits provide little additional performance in such cases. The exception is an isolation platform where the system may be lightly damped in order to achieve a faster local rolloff after the resonance. As another consideration, the outcome of resonant shunt circuits, to introduce additional damping and reduce the relative plunger velocity, may not be desirable. In both inertial vibration control and isolation columns, the opposite is desired, a lightly damped resonance is required to counteract the structural vibration of the system. It may actually be necessary to reduce the system damping. For these reasons, the only techniques discussed in detail, are those capable of minimizing an arbitrary performance objective, one that can be applied in any situation. 


\section{B. Ideal Negative-Inductor Controller}

By substituting the equivalent impedance parameterized feedback controller (11) into the closed-loop transfer function (9), we obtain

$$
\frac{\nu(s)}{I_{1}(s)}=\frac{G_{\nu F}(s) C_{1}(L s+R+Z(s))}{L s+R+Z(s)+C_{3} C_{4} G_{\nu F}(s)} .
$$

Observe that the numerator is affine in the transfer function $L s+$ $R+Z(s)$. Thus, by equating $L s+R+Z(s)$ to zero, the closedloop transfer function $\nu(s) / I_{1}(s)$ can be reduced to zero. In other words, by choosing

$$
Z(s)=-L s-R
$$

or

$$
Y(s)=\frac{1}{-L s-R}
$$

any introduced disturbance will have little or no effect on the mechanical system. A similar result exists for piezoelectric transducers [19].

As is the case for piezoelectric transducers, and as one might expect, such a "miracle" controller has limited practical use. By implementing (13) or (14) the equivalent velocity feedback controller is simply a proportional controller of infinite gain. Besides the magnitude of control energy required, the stability and performance is extremely sensitive to small changes in the transducer dynamics.

In practice, by tuning the magnitudes of the negative inductor and resistor, the control effort can be toned down. Due to the ad hoc nature of this approach, it is difficult to accurately manipulate the tradeoff between control effort and damping performance. For example, using a negative inductor-resistor controller, it is impossible to distribute, concentrate, or mitigate the control energy associated with individual structural modes. Neither is it possible to minimize a specific performance function not proportionally related to the plunger velocity. In cases where the goal is not simply to reduce the magnitude of plunger velocity, such as in acoustic, isolation, and suspension systems, the negative inductor controller is of little use.

In spite of the associated problems, this technique warrants mention due to its inherent simplicity and utility in gaining an intuitive understanding of the abstract controllers generated from an automated synthesis process such as LQR.

\section{Impedance Synthesis}

Referring to Fig. 8, the shunted electromechanical system can be regarded as a typical feedback control system where a disturbance $I_{1}$ results in a vibration characterized by the velocity $\nu$.

In order to apply standard synthesis techniques such as LQR, we require a minimal state-space model representing the composite system. By defining the following state-space model for the coil admittance $1 /(L s+R)$ :

$$
\begin{aligned}
\dot{x}_{y}(t) & =\mathbf{A}_{y} x_{y}(t)+\mathbf{B}_{y} V(t) \\
I_{z}(t) & =\mathbf{C}_{y} x_{y}(t)
\end{aligned}
$$

where, $\mathbf{A}_{y}=[-(R / L)], \mathbf{B}_{y}=[1]$, and $\mathbf{C}_{y}=[1 / L]$, the following state-space model is easily derived for the composite system $G$ :

$$
\begin{aligned}
\dot{x}_{g}(t) & =\mathbf{A}_{g} x_{g}(t)+\mathbf{B}_{g}\left[\begin{array}{l}
I_{1}(t) \\
V_{z}(t)
\end{array}\right] \\
{\left[\begin{array}{c}
\dot{x}(t) \\
I_{z}(t)
\end{array}\right] } & =\mathbf{C}_{g} x_{g}(t)
\end{aligned}
$$

where

$$
x_{g}(t)=\left[\begin{array}{l}
x_{p}(t) \\
x_{y}(t)
\end{array}\right] \quad \mathbf{B}_{g}=\left[\begin{array}{cc}
\mathbf{B}_{p} C_{1} C_{4} & \mathbf{0} \\
\mathbf{0} & -\mathbf{B}_{y}
\end{array}\right]
$$

and

$$
\mathbf{A}_{g}=\left[\begin{array}{cc}
\mathbf{A}_{p} & \mathbf{B}_{p} \mathbf{C}_{y} C_{3} C_{4} \\
\mathbf{B}_{y} \mathbf{C}_{p} & \mathbf{A}_{y}
\end{array}\right] \quad \mathbf{C}_{g}=\left[\begin{array}{cc}
\frac{1}{C_{4}} \mathbf{C}_{p} & \mathbf{0} \\
\mathbf{0} & \mathbf{C}_{y}
\end{array}\right]
$$

Our design objective is to minimize the velocity $\nu$ while restraining the magnitude of the control signal $V_{z}$. In a linear quadratic sense, the objective is to minimize

$$
J=\int_{-\infty}^{\infty}\left[\nu^{2}(t)+\left(k_{u} V_{z}(t)\right)^{2}\right] d t
$$

where $k_{u}$ is the control signal weighting. Restated, in the standard LQR context

$$
J=\int_{-\infty}^{\infty}\left[x_{g}^{\prime}(t) Q x_{g}(t)+u^{\prime}(t) R u(t)\right] d t
$$

the corresponding $Q$ and $R$ matrices are $Q=$ $\left[\begin{array}{ll}\left(1 / C_{4}\right) \mathbf{C}_{p} & \mathbf{0}\end{array}\right]^{\prime}\left[\left(1 / C_{4}\right) \mathbf{C}_{p} \mathbf{0}\right]$, and $R=k_{u}^{2}$.

We can also consider the $\mathcal{H}_{2}$ control objective where we seek to minimize, in the $\mathcal{H}_{2}$ sense, the weighted sum of velocity and the control signal in response to a specific disturbance $I_{1}$, i.e., we seek to minimize

$$
J=\left\|\frac{\nu(s)+k_{u} V_{z}(s)}{I_{1}(s)}\right\|_{2} .
$$

This specification is easily cast as a standard $\mathcal{H}_{2}$ problem by considering the modified plant $\tilde{G}$ shown in Fig. 10. Minimizing (19) is now equivalent to minimizing

$$
J=\left\|\frac{z(s)}{w(s)}\right\|_{2}
$$

where the modified plant model is that of (16) with a nonzero $D$ matrix

$$
\tilde{\mathbf{D}}_{g}=\left[\begin{array}{cc}
\mathbf{0} & k_{u} \\
\mathbf{0} & \mathbf{0}
\end{array}\right]
$$

\section{Admittance Synthesis}

Referring to Fig. 7, the shunted electromechanical system can be regarded as a typical feedback control system where a disturbance $I_{1}$ results in a vibration characterized by the velocity $\nu$. 


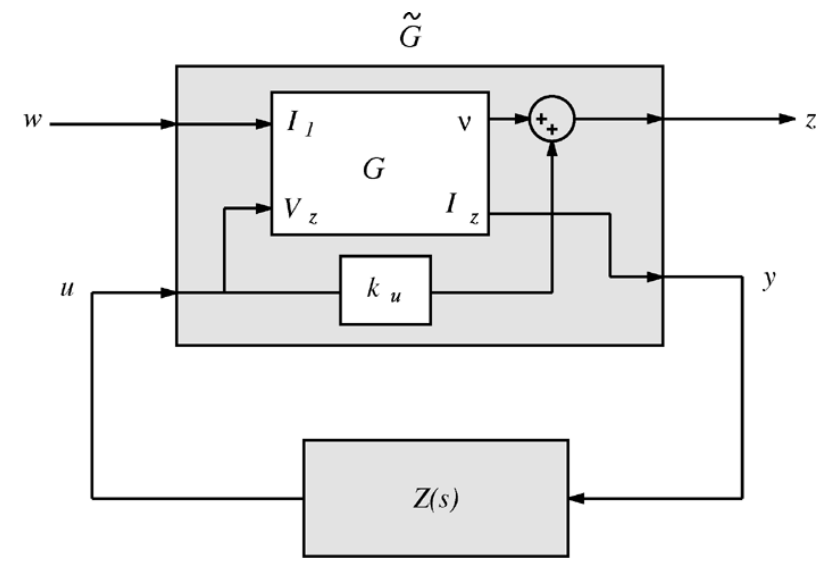

Fig. 10. Modified plant model required for $\mathcal{H}_{2}$ impedance synthesis.

(a)

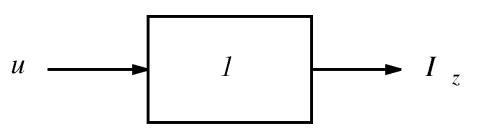

(b)

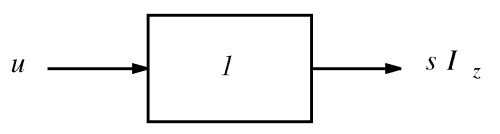

(c) $u \longrightarrow \frac{s}{L_{s} s+R}{ }_{s} \longrightarrow s I_{z}$

Fig. 11. (a) Unity gain current amplifier. (b) Unity gain current fluxion amplifier. (c) Current fluxion amplifier with internal dynamics.

Due to the improper transfer function from the applied current $I_{z}$ to the voltage $V_{z}$, we cannot directly apply, as in Section III-C, the standard state-space design methodologies. To overcome this difficulty, we introduce the notion of a current fluxion amplifier.

As opposed to a regular current amplifier that drives an output current proportional to the applied reference signal, a current fluxion amplifier, as its name suggests, drives a current-rate-ofchange proportional to the applied reference signal. Fig. 11(a) and (b), compare the operation of a current and current fluxion amplifier. Where the current amplifier in Fig. 11(a) has a gain of $1 \mathrm{~A} / \mathrm{V}$, the current fluxion amplifier in Fig. 11(b) has a gain of $1 \mathrm{As}^{-1} / \mathrm{V}$. Such an amplifier, when connected to an electromagnetic coil, can also be viewed as a self-induced-voltage amplifier, the voltage drop across the transducer's self inductance is proportional to the derivative of current.

In practice, to avoid the large low-frequency gains associated with integration, the current fluxion amplifier is best implemented by controlling the voltage across a sensing inductance $L_{s}$ and resistance $R_{s}$. In this case, the current fluxion amplifier includes some internal dynamics as shown in Fig. 11(c). The resistance results in a limited gain at low frequencies. More details on the practical implementation of a current fluxion amplifier can be found in Section IV-B.

When dealing with a current fluxion driven transducer, the modified composite system is as shown in Fig. 12(a). By concatenating the transfer functions of coil impedance and amplifier dynamics, the resulting generalized plant model as shown in Fig. 12(b), is proper. (a)

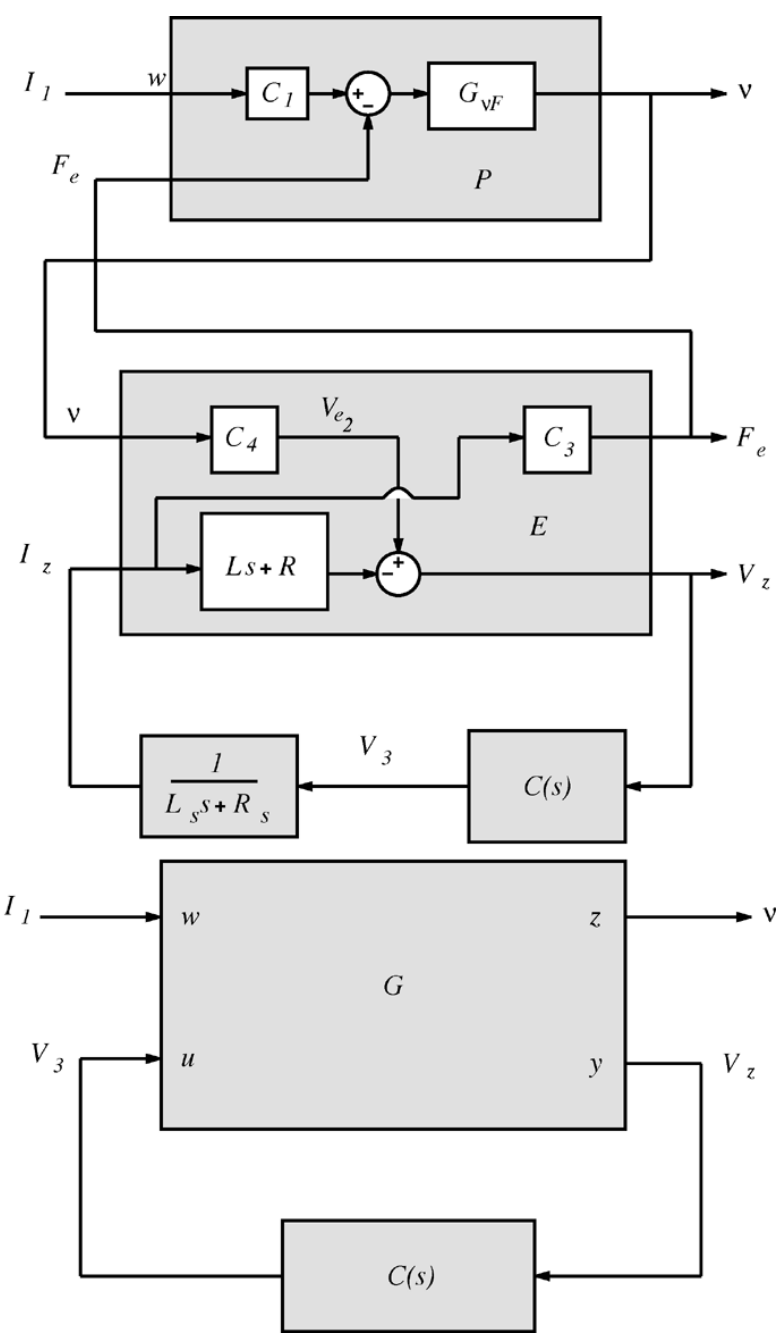

Fig. 12. (a) Electromechanical system including the dynamics of a current fluxion amplifier. (b) In generalized plant/controller form.

Inadvertently, by solving the problem associated with the improper coil impedance, we have also achieved a better conditioning of the transfer function from the command input to $V_{z}$. The dynamic range of this response was previously dominated by the coil impedance, but when driving the coil with current fluxion rather than current, the dynamic range is almost solely dependent on the mechanical response. As will become clearer in Section IV, this characteristic affords a more accurate estimation of the structural state.

In order to apply standard synthesis techniques such as LQR, we require a minimal state-space model representing the current fluxion driven composite system. By defining the following state-space model for the concatenated coil impedance and amplifier dynamics $(L s+R) /\left(L_{s} s+R_{s}\right)$

$$
\begin{aligned}
\dot{x}_{z}(t) & =\mathbf{A}_{z} x_{z}(t)+\mathbf{B}_{z} V_{3}(t) \\
{\left[\begin{array}{c}
V_{c}(t) \\
I_{z}(t)
\end{array}\right] } & =\left[\begin{array}{l}
\mathbf{C}_{z 1} \\
\mathbf{C}_{z 2}
\end{array}\right] x_{z}(t)+\left[\begin{array}{c}
\mathbf{D}_{z 1} \\
\mathbf{0}
\end{array}\right] V_{3}(t)
\end{aligned}
$$

where, $\mathbf{A}_{z}=\left[-\left(R_{s} / L_{s}\right)\right], \mathbf{B}_{z}=[1], \mathbf{C}_{z 1}=\left[\left(R / L_{s}\right)-\right.$ $\left.\left(R_{s} L / L_{s}^{2}\right)\right], \mathbf{C}_{z 2}=\left[1 / L_{s}\right], \mathbf{D}_{z 1}=\left[L / L_{s}\right]$, a state-space 


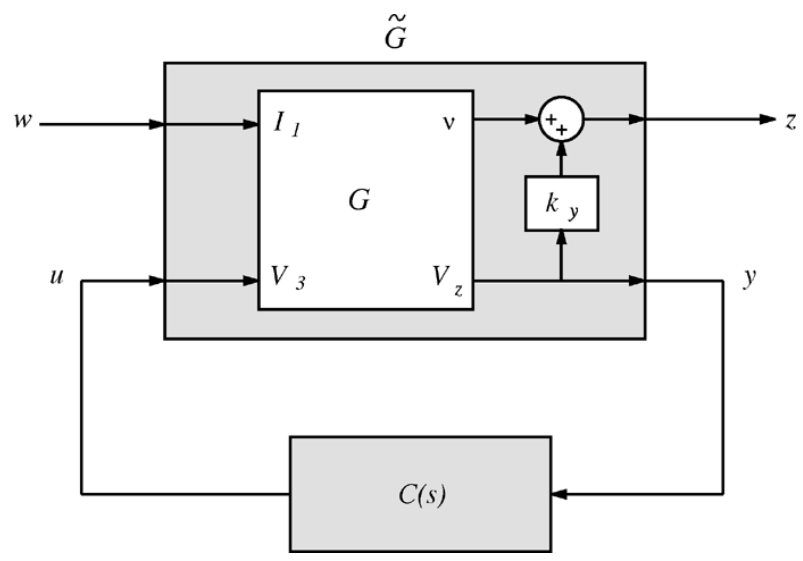

Fig. 13. Modified plant model required for admittance synthesis.

model can be derived for the composite system $G . V_{c}$ is the voltage dropped across the internal impedance of the transducer

$$
\begin{gathered}
\dot{x}_{g}(t)=\mathbf{A}_{g} x_{g}(t)+\mathbf{B}_{g}\left[\begin{array}{l}
I_{1}(t) \\
V_{3}(t)
\end{array}\right] \\
{\left[\begin{array}{c}
\dot{x}(t) \\
V_{z}(t)
\end{array}\right]=\mathbf{C}_{g} x_{g}(t)+\mathbf{D}_{g}\left[\begin{array}{l}
I_{1}(t) \\
V_{3}(t)
\end{array}\right]}
\end{gathered}
$$

where

$$
\begin{aligned}
x_{g}(t) & =\left[\begin{array}{l}
x_{p}(t) \\
x_{z}(t)
\end{array}\right] \\
\mathbf{B}_{g} & =\left[\begin{array}{cc}
\mathbf{B}_{p} C_{1} C_{4} & \mathbf{0} \\
\mathbf{0} & \mathbf{B}_{z}
\end{array}\right] \\
\mathbf{D}_{g} & =\left[\begin{array}{cc}
\mathbf{0} & \mathbf{0} \\
\mathbf{0} & -\mathbf{D} \\
z 1
\end{array}\right]
\end{aligned}
$$

and

$$
\mathbf{A}_{g}=\left[\begin{array}{cc}
\mathbf{A}_{p} & -\mathbf{B}_{p} \mathbf{C}_{z 2} C_{3} C_{4} \\
\mathbf{0} & \mathbf{A}_{z}
\end{array}\right] \quad \mathbf{C}_{g}=\left[\begin{array}{cc}
\mathbf{C}_{p} \frac{1}{C_{4}} & \mathbf{0} \\
\mathbf{C}_{p} & -\mathbf{C}_{z 1}
\end{array}\right] .
$$

Our design objective is to minimize the velocity $\nu$, whilst, as opposed to restraining the control signal $V_{3}$, it remains of interest to limit the magnitude of the terminal voltage $V_{z}$. In a linear quadratic sense, the objective is to minimize

$$
J=\int_{-\infty}^{\infty}\left[\nu^{2}(t)+\left(k_{y} V_{z}(t)\right)^{2}\right] d t
$$

where $k_{y}$ is the weighting on the terminal voltage $V_{z}$. This specification can be cast as a standard LQR problem by considering the modified plant $\tilde{G}$ shown in Fig. 13. The state-space representation of which is

$$
\begin{aligned}
\dot{x}_{g}(t) & =\mathbf{A}_{g} x_{g}(t)+\mathbf{B}_{g}\left[\begin{array}{l}
w(t) \\
u(t)
\end{array}\right] \\
{\left[\begin{array}{l}
z(t) \\
y(t)
\end{array}\right] } & =\tilde{\mathbf{C}}_{g} x_{g}(t)+\tilde{\mathbf{D}}_{g}\left[\begin{array}{l}
w(t) \\
u(t)
\end{array}\right]
\end{aligned}
$$

where

$$
\begin{aligned}
& \tilde{\mathbf{C}}_{g}=\left[\begin{array}{c}
\tilde{\mathbf{C}}_{g 1} \\
\tilde{\mathbf{C}}_{g 2}
\end{array}\right]=\left[\begin{array}{cc}
{\left[\begin{array}{cc}
\mathbf{C}_{p} \frac{1}{C_{4}} & \mathbf{0}
\end{array}\right]+k_{y}\left[\begin{array}{ll}
\mathbf{C}_{p} & -\mathbf{C}_{z 1}
\end{array}\right]} \\
{\left[\begin{array}{lll}
\mathbf{C}_{p} & -\mathbf{C}_{z 1}
\end{array}\right]}
\end{array}\right]
\end{aligned}
$$

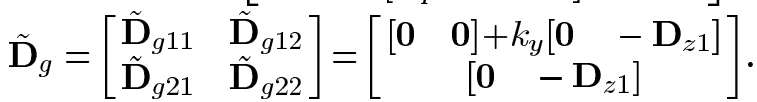

Minimizing (28) is now equivalent to minimizing

$$
\begin{aligned}
& J= \int_{-\infty}^{\infty} z^{2}(t) d t \\
&=\int_{-\infty}^{\infty}\left[\tilde{\mathbf{C}}_{g 1} x_{g}(t)+\tilde{\mathbf{D}}_{g 12} u(t)\right]^{\prime} \\
& \quad \times\left[\tilde{\mathbf{C}}_{g 1} x_{g}(t)+\tilde{\mathbf{D}}_{g 12} u(t)\right] d t \\
&=\int_{-\infty}^{\infty}\left[x_{g}^{\prime}(t) \tilde{\mathbf{C}}_{g 1}^{\prime} \tilde{\mathbf{C}}_{g 1} x_{g}(t)+u^{\prime}(t) \tilde{\mathbf{D}}_{g 12}^{\prime} \tilde{\mathbf{D}}_{g 12} u(t)\right. \\
&\left.\quad+2 x_{g}^{\prime}(t) \tilde{\mathbf{C}}_{g 1}^{\prime} \tilde{\mathbf{D}}_{g 12} u(t)\right] d t .
\end{aligned}
$$

Restated, in the standard LQR context

$$
J=\int_{-\infty}^{\infty}\left[x_{g}^{\prime}(t) Q x_{g}(t)+u^{\prime}(t) R u(t)+x_{g}^{\prime}(t) N u(t)\right] d t
$$

The corresponding $Q, R$, and $N$ matrices are

$$
\begin{aligned}
Q & =\tilde{\mathbf{C}}_{g 1}^{\prime} \tilde{\mathbf{C}}_{g 1} \\
R & =\tilde{\mathbf{D}}_{g 12}^{\prime} \tilde{\mathbf{D}}_{g 12} \\
N & =\tilde{\mathbf{C}}_{g 1}^{\prime} \tilde{\mathbf{C}}_{g 12} .
\end{aligned}
$$

We can also consider the $\mathcal{H}_{2}$ control objective where we seek to minimize, in the $\mathcal{H}_{2}$ sense, the weighted sum of velocity and the terminal voltage in response to a specific disturbance $I_{1}$, i.e., we seek to minimize

$$
J=\left\|\frac{\nu(s)+k_{y} V_{z}(s)}{I_{1}(s)}\right\|_{2} .
$$

By considering the modified plant $\tilde{G}$ described in (29), this specification is easily cast into a standard $\mathcal{H}_{2}$ synthesis problem. Minimizing (36) is now equivalent to minimizing

$$
J=\left\|\frac{z(s)}{w(s)}\right\|_{2} .
$$

\section{EXPERIMENTAL RESULTS}

To verify the modeling and design techniques presented in preceding sections, each method has been applied to an experimental electromechanical system. 


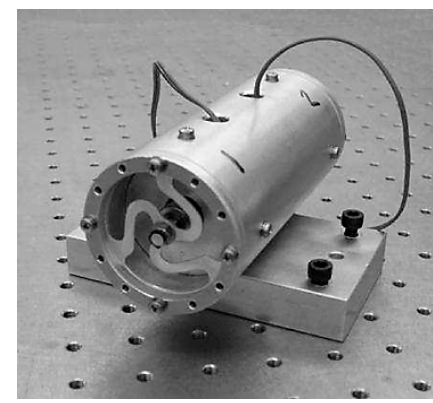

Fig. 14. External photograph of the experimental electromagnetic apparatus.

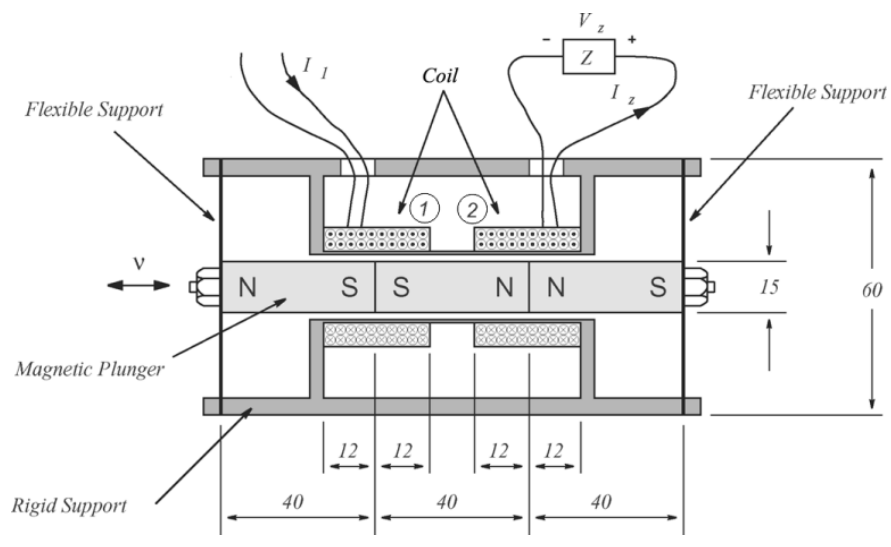

Fig. 15. Side section of the experimental electromagnetic apparatus. All dimensions are in millimeters.

\section{A. Electromagnetic Transducer}

A photograph of the electromagnetic transducer showing the rigid body, flexible end supports, mounting plate, and coils is provided in Fig. 14. The apparatus is essentially a translational solenoid with two identical fixed coils and a magnetic plunger supported at either end by flexible disks. A side section including dimensions and magnetic orientations is shown in Fig. 15.

The coils are wound from $0.25 \mathrm{~mm}$ diameter enamel coated copper wire and have an electrical impedance of $3.3 \Omega+1 \mathrm{mH}$. In order to prevent distortion of the magnetic flux field, only nonmagnetic materials, such as aluminum and copper, were used in the construction of the rigid body, flexible end supports and mounting plate.

In practice, the magnetic field strength, as well as being a function of the magnetic material, is limited by the maximum allowable dimensions and weight of the magnets. In these experiments, three rare earth magnets (Neodymium Iron Boron), are arranged to form the magnetic plunger as shown in Fig. 15. At the two points where opposing poles meet (at the center of each winding), a strong magnetic field exits at right angles to the plunger. When the plunger is in motion, the strong parallel field flowing through the coil results in a high-flux density and correspondingly large induced voltage. The physical parameters of the electromagnetic and mechanical systems are summarized in Table I.

The plunger velocity is measured using a PSV-300 Polytec Scanning Laser Vibrometer.
TABLE I

ELECTROMECHANICAL SYSTEM PARAMETERS

\begin{tabular}{l|l}
\hline Parameter & Value \\
\hline Spring constant $K$ & $56 \mathrm{kNm}^{-1}$ \\
\hline Damping coefficient $C$ & $2.667 \mathrm{Nsm}^{-1}$ \\
\hline Plunger mass $M$ & $0.150 \mathrm{~kg}$ \\
\hline Electromagnetic Coupling $C_{1}$ & 3.55 \\
\hline Electromagnetic Coupling $C_{2}$ & 4.06 \\
\hline Electromagnetic Coupling $C_{3}$ & 3.55 \\
\hline Electromagnetic Coupling $C_{4}$ & 4.06 \\
\hline Coil Inductance $L$ & $1 \mathrm{mH}$ \\
\hline Coil Resistance $R$ & $3.3 \Omega$ \\
\hline
\end{tabular}
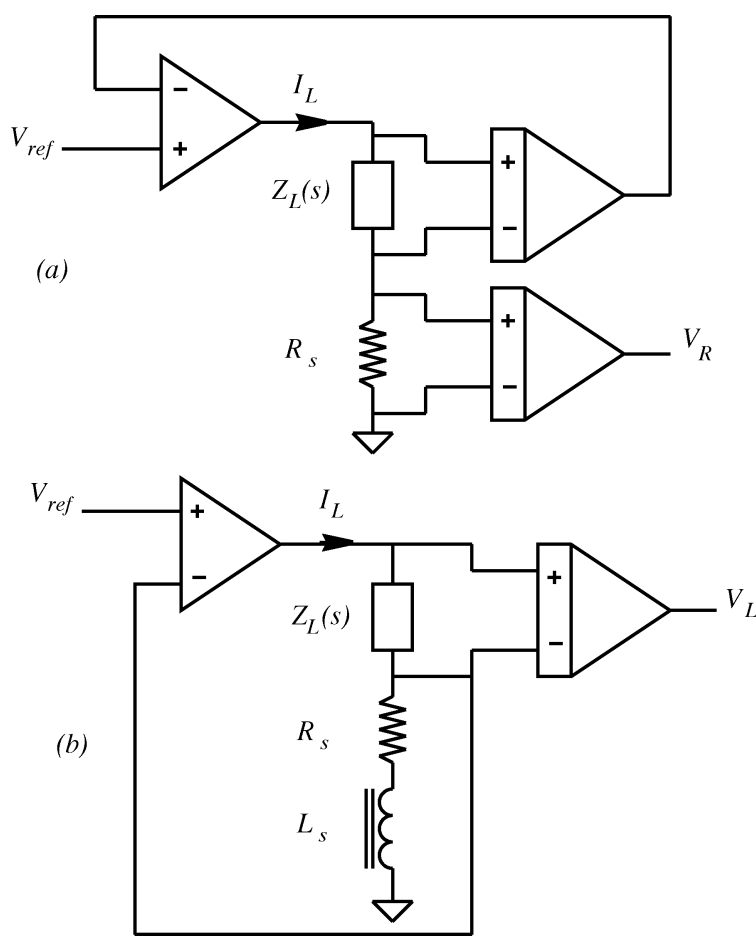

Fig. 16. Simplified schematic of (a) differential voltage feedback amplifier and (b) current fluxion feedback amplifier.

\section{B. Power Amplifier and Instrumentation}

In order to implement the arbitrary shunt impedance and admittances resulting from control design, a power amplifier was developed capable of driving either differential terminal voltages or load current fluxions. During either mode of operation, the device is also capable of instrumenting the respective load current or terminal voltage.

The simplified schematic of two circuits realizing each mode of operation are shown in Fig. 16(a) and (b). Both circuits incorporate a high-gain feedback loop to control either the measured load or sensing impedance voltage.

In Fig. 16(a), within the high-frequency bandwidth of the control loop, the reference potential $V_{\text {ref }}$ appears across the load, i.e., we have a unity gain voltage amplifier. The additional resistance and differential amplifier generate the current measurement $V_{R}$ with gain $R_{s} V / A$.

In Fig. 16(b), within the high-frequency bandwidth of the control loop, the reference potential $V_{\text {ref }}$ appears across the 


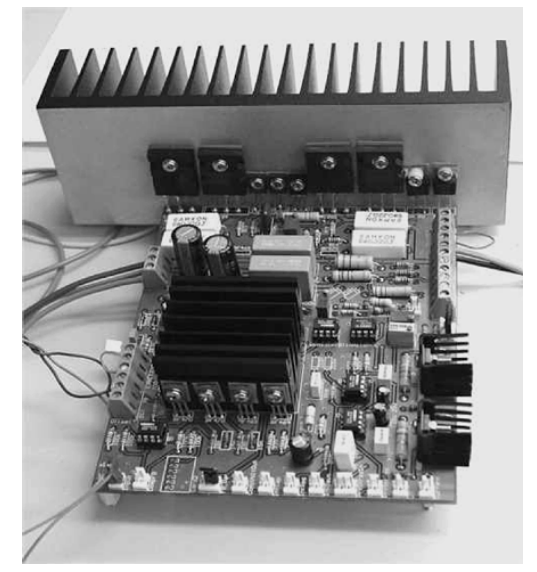

Fig. 17. Implementation of a voltage and current fluxion amplifier.

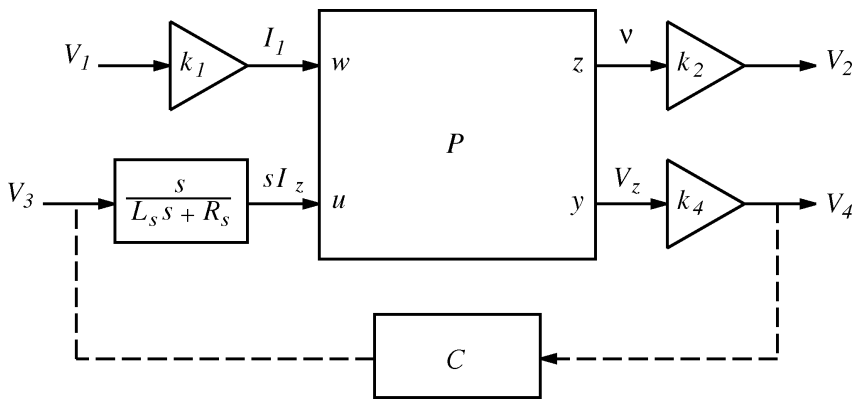

Fig. 18. Open-loop external gains of the shunt voltage controlled electromagnetic system.

sensing impedance $L_{s} s+R_{s}$, thus, the resulting load current is described by

$$
\begin{aligned}
\frac{I_{L}(s)}{V_{\text {ref }}(s)} & =\frac{1}{L_{s} s+R_{s}} \\
& \approx \frac{1}{L_{s} s} .
\end{aligned}
$$

Neglecting the resistance $R_{s}$, the amplifier can be thought of as an integrator connected in series to the reference of a current source (with gain $1 / L_{s} \mathrm{~A} / \mathrm{V}$ ). Alternatively, as it is defined in this paper, we could also refer to the device as a current fluxion amplifier (with gain $\left(1 / L_{s}\right) \mathrm{As}^{-1} / \mathrm{V}$ ) where the reference signal $V_{\text {ref }}$ commands the rate of change in load current. The parasitic coil resistance $R_{s}$ is beneficial and effectively limits the low-frequency gain of the amplifier eliminating the normal problems associated with integration. The following in-bandwidth transfer function of the current fluxion amplifier is determined by the parameters of the sensing inductor

$$
\frac{s I_{L}(s)}{V_{r e f}(s)}=\frac{s}{L_{s} s+R_{s}} .
$$

A practical implementation of a combination voltage and current fluxion amplifier is shown in Fig. 17. The device is capable of \pm 250 - $\mathrm{V}$ operation at a maximum dc current of $32 \mathrm{~A}$.

A dSpace 1005 based system is used to implement the required impedance and admittance transfer functions.
TABLE II

External Gains Associated With the Shunt Voltage Controlled ELECTROMAGNETIC SYSTEM

\begin{tabular}{l|l}
\hline Gain & Value \\
\hline$k_{1}$ & $1 \mathrm{~A} / \mathrm{V}$ \\
\hline$k_{2}$ & $40 \mathrm{~V} / \mathrm{ms}^{-1}$ \\
\hline$k_{3}$ & $-4 \mathrm{~V} / \mathrm{V}$ \\
\hline$k_{4}$ & $10 \mathrm{~V} / \mathrm{A}$ \\
\hline
\end{tabular}
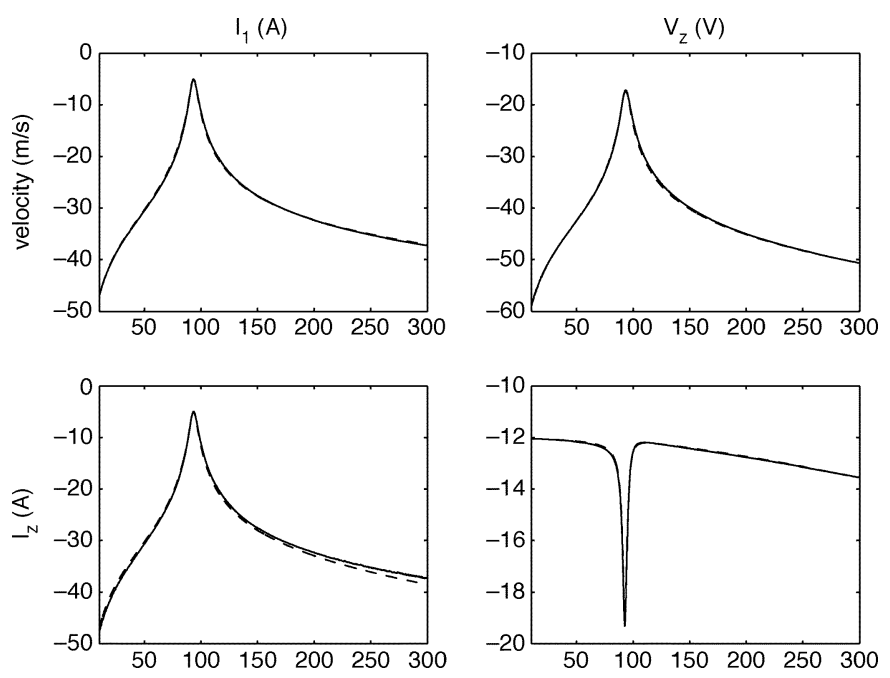

Fig. 19. Simulated (-) and experimental (- -) magnitude frequency response (in decibels) of the shunt voltage controlled electromagnetic system.

\section{Impedance Synthesis}

Fig. 18 shows the instrumentation and driver gains associated with the underlying electromechanical system. The voltages $V_{1}$ through $V_{4}$ represent the signals applied to, or measured from, the power amplifiers and instrumentation. The gain and units associated with each signal can be found in Table II. The actual electrical shunt impedance presented to the coil is related to the controller through the gains $k_{3}$ and $k_{4}$, specifically

$$
Z_{c}(s)=\frac{V_{z}(s)}{I_{z}(s)}=k_{3} C(s) k_{4} .
$$

To assess the accuracy of the analytic model (discussed in Section II-C), the simulated frequency response is compared to that measured directly from the experimental system. A multivariable frequency response is measured successively from each input to output pair. During the component single-input-singleoutput (SISO) frequency response measurements, the residual input is set to zero. The magnitude and phase frequency responses are shown, respectively, in Figs. 19 and 20. In the frequency domain, a good correlation can be observed between the analytic model and measured system.

1) LQR Impedance Synthesis: As discussed in Section III-C, a linear quadratic regulator can be designed to command the shunt terminal voltage $V_{z}$ with a view to regulating a performance signal consisting of the weighted sum of plunger velocity and control signal. An observer is required to estimate the system states from the measured shunt current $I_{z}$. Once designed, the concatenation of observer and LQR gain matrix results in a system, interpreted as an active shunt 

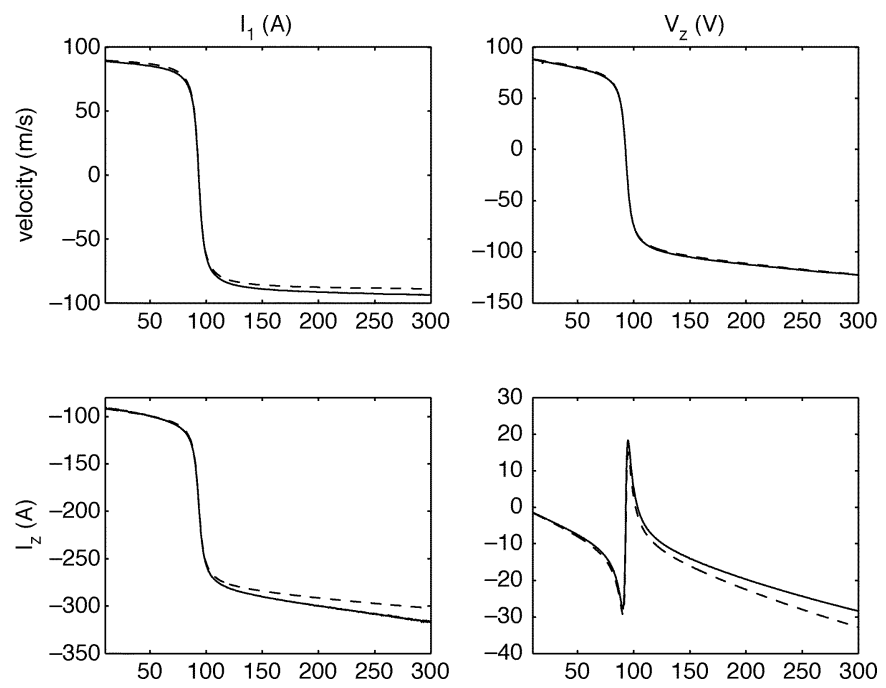

Fig. 20. Simulated (-) and experimental (- -) phase frequency response (in degrees) of the shunt voltage controlled electromagnetic system.
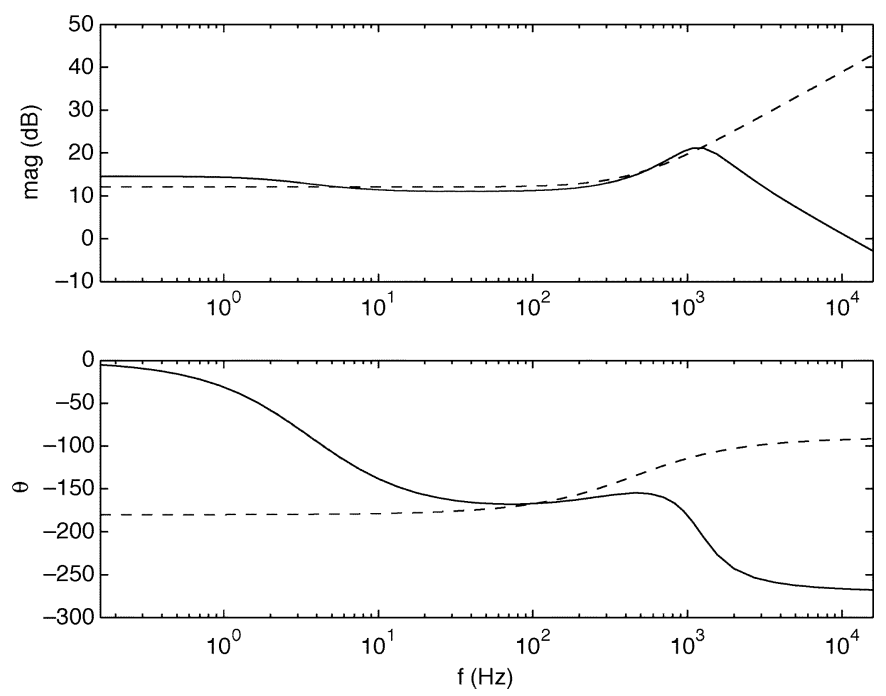

Fig. 21. Complex impedance of the LQR (-) and ideal negative inductor-resistor controller (- -).

impedance, that can be applied to one of the electromagnetic coils in order to reduce structural vibration.

Based on the physical model (including external gains) that was validated in the previous subsection, and referring to the notation introduced in Section III-C, an LQR gain matrix was designed to minimize the following performance function:

$$
J=\int_{-\infty}^{\infty}\left[\left(k_{2} \nu(t)\right)^{2}+\left(\frac{7}{k_{3}} V_{z}(t)\right)^{2}\right] d t
$$

where the factor 7 represents the relative control weighting. The gains $k_{2}$ and $k_{3}$ are included as the design is based on the input-output model which includes the amplifier and instrumentation dynamics. The observer was designed by pole placement, where the target poles were chosen as that of the closed-loop system with real components multiplied by 2 . As is routine in control system design, the control weighting of 7 and observer pole locations were chosen experimentally to achieve a reasonable tradeoff between damping performance, robustness, and the control signal magnitude.

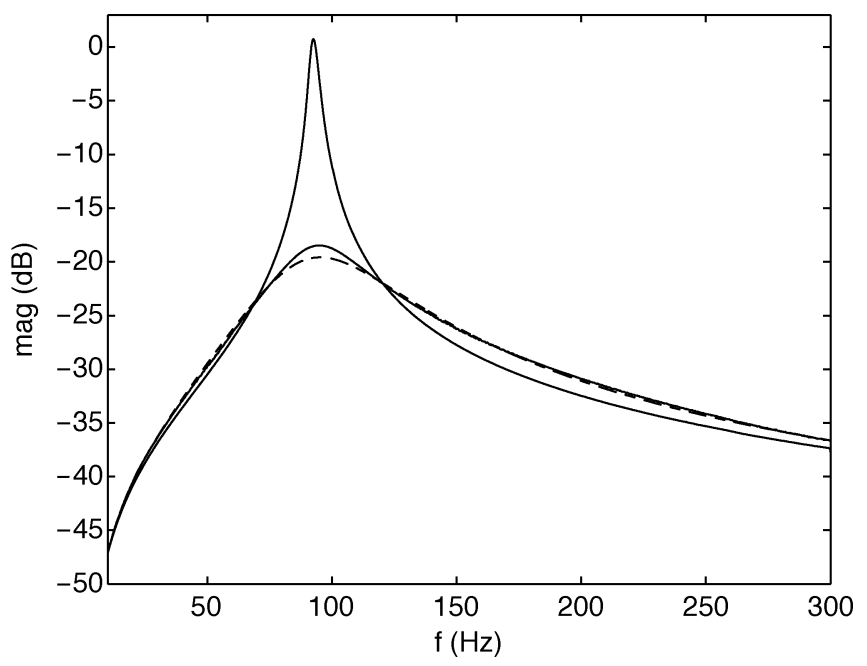

Fig. 22. Experimental (-) and simulated (- -) open- and closed-loop frequency response from an applied disturbance current $I_{1}(\mathrm{~A})$ to the resulting plunger velocity $\nu\left(\mathrm{ms}^{-1}\right)$ for the LQR impedance controlled system. The open-loop frequency response is also shown (-).
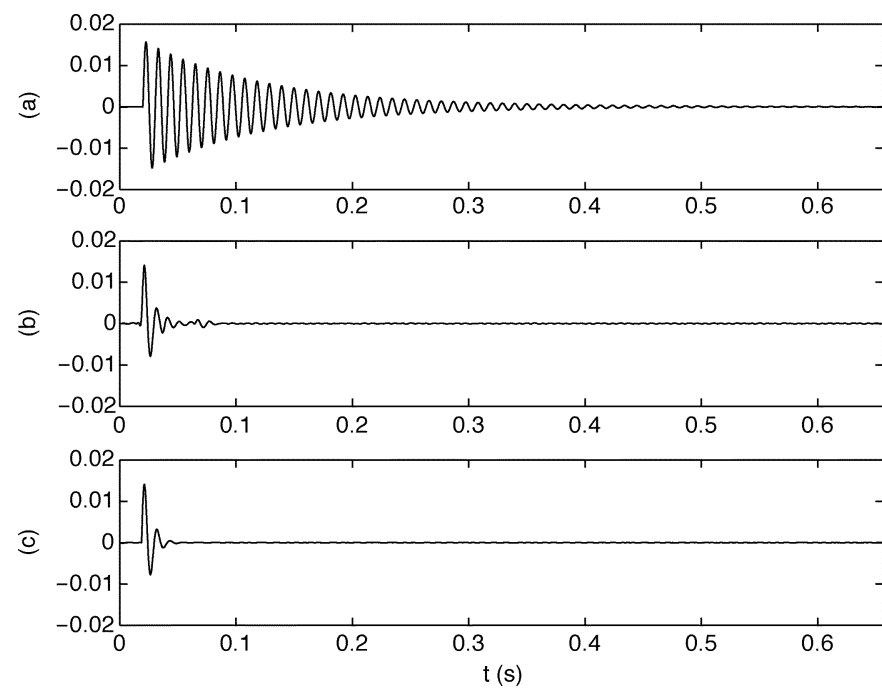

Fig. 23. Velocity response $\nu\left(\mathrm{ms}^{-1}\right)$ of the LQR impedance controlled system to a step disturbance current $I_{1}$. (a) Experimental open loop, (b) closed loop (b), and (c) simulated closed loop.

The complex impedances of the implemented LQR and ideal negative inductor-resistor controller are plotted in Fig. 21. It can be observed that at frequencies close to the resonance of the system, $92 \mathrm{~Hz}$, the impedance of the LQR designed controller closely resembles that of the ideal negative inductor-resistor controller. Beneficially, the LQR designed impedance is proper, has a limited bandwidth, and exerts control effort only at frequencies close to the resonance of the system (which can be deduced from the following closed-loop performance plots).

The damping performance of the LQR controller was assessed in both the frequency and time domains. With the controller in the loop, a disturbance current $I_{1}$, proportional to a force disturbance, is applied to the system. Experimental and simulated open- and closed-loop frequency responses are shown in Fig. 22. The controller was measured to reduce the resonant peak by $19.4 \mathrm{~dB}$. The corresponding time domain velocity response to a $300 \mathrm{~Hz}$ low-pass filtered step change in disturbance current $I_{1}$ is shown in Fig. 23. The simulated 


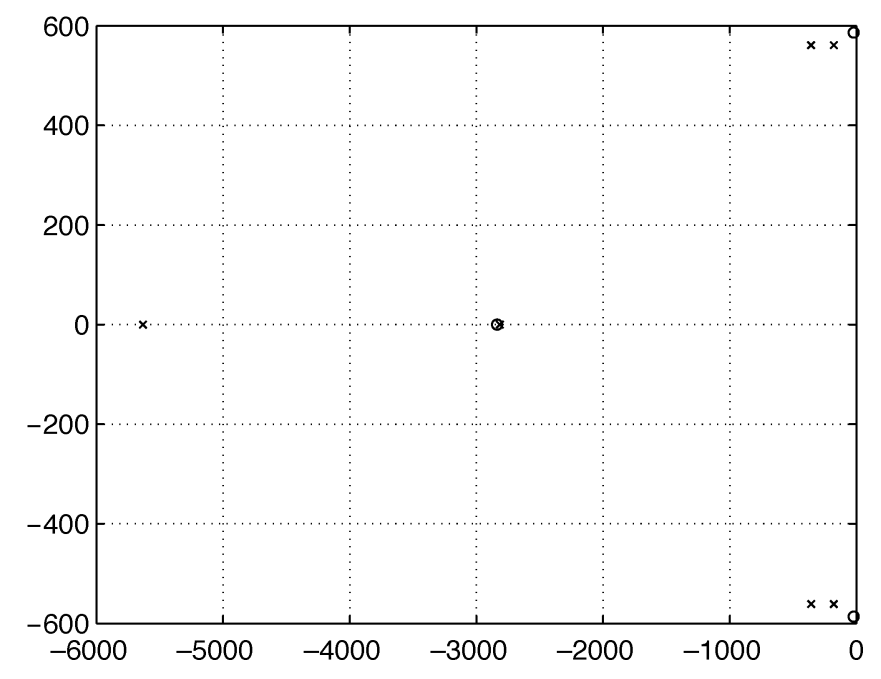

Fig. 24. Open- $(\bigcirc)$ and closed-loop $(x)$ pole locations of the LQR impedance controlled system.

closed-loop step response was obtained by recording the applied step signal and applying it in simulation to the closed-loop model. The action of the controller can be further understood by observing the open- and closed-loop pole locations shown in Fig. 24. Disregarding the observer poles, the controller has acted to increase system damping by shifting the associated poles further into the left half plane. The pole corresponding to the coil admittance remains effectively unchanged.

2) $\mathcal{H}_{2}$ Impedance Synthesis: In analogy to Section IV-C1, and as discussed in Section IV-C2, this subsection documents the implementation of an active shunt impedance designed to minimize the $\mathcal{H}_{2}$ norm of the transfer function between a disturbance current $I_{1}$ and a performance signal $z$. As in Section IV-C1, the performance signal consists of the weighted sum of plunger velocity and control signal. Specifically, for the electromagnetic system including external gains, we seek to minimize

$$
J=\left\|\frac{k_{2} \nu(s)+\frac{k_{u}}{k_{3}} V_{z}(s)}{\frac{I_{1}(s)}{k_{1}}}\right\|_{2}
$$

where $k_{u}$ is the control signal weighting.

For the plant under consideration, the $\mathcal{H}_{2}$ problem is well defined and feasible. All of the standard requirements are met, i.e., the plant is minimal, proper, controllable, observable, and of finite dimension. However, in order to find a solution using existing tools, i.e., the algebraic Riccati solution implemented by the $\mu$-Synthesis Toolbox for Matlab, the system must meet some additional requirements. The most problematic of which, is the requisite full rank condition on the standard plant matrices $D_{21}$ and $D_{12}$. In this case, where each of the signals $w$, $u, y$, and $z$ are unidimensional, this condition requires that the feedthrough term from $w$ to $y$, and $u$ to $z$, is nonzero. As the performance signal $z$ already contains a direct weighting on the control signal $V_{z}$, the only condition not met is that on $D_{21}$. To overcome this problem, for the purpose of controller synthesis, we include an artificial feedthrough term $D_{21}$. We now have two design parameters: $k_{u}$ and $D_{21}$. These were chosen to be 0.1
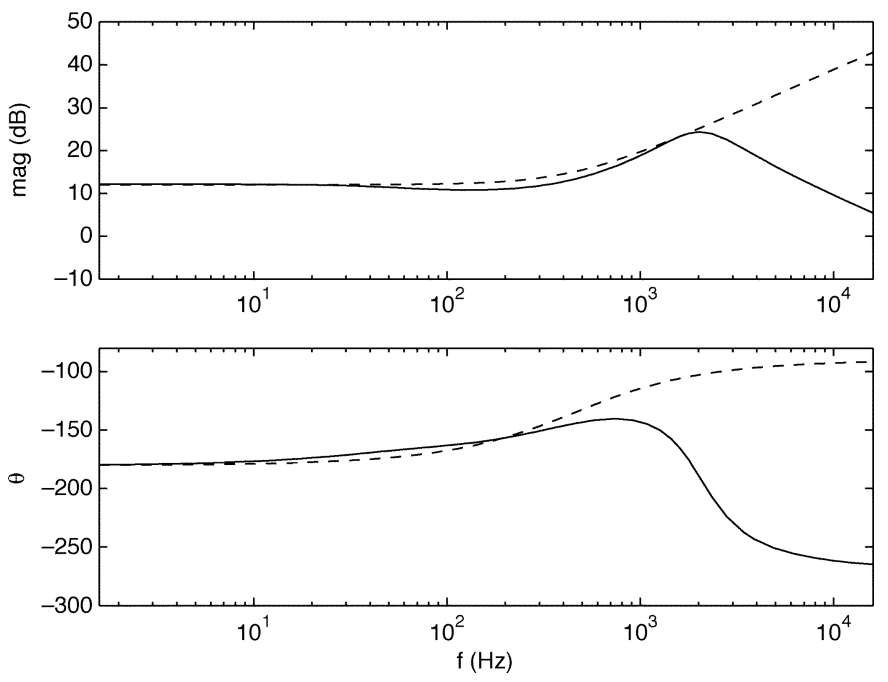

Fig. 25. Complex impedance of the $\mathcal{H}_{2}(-)$ and ideal negative inductor-resistor controller (- -).

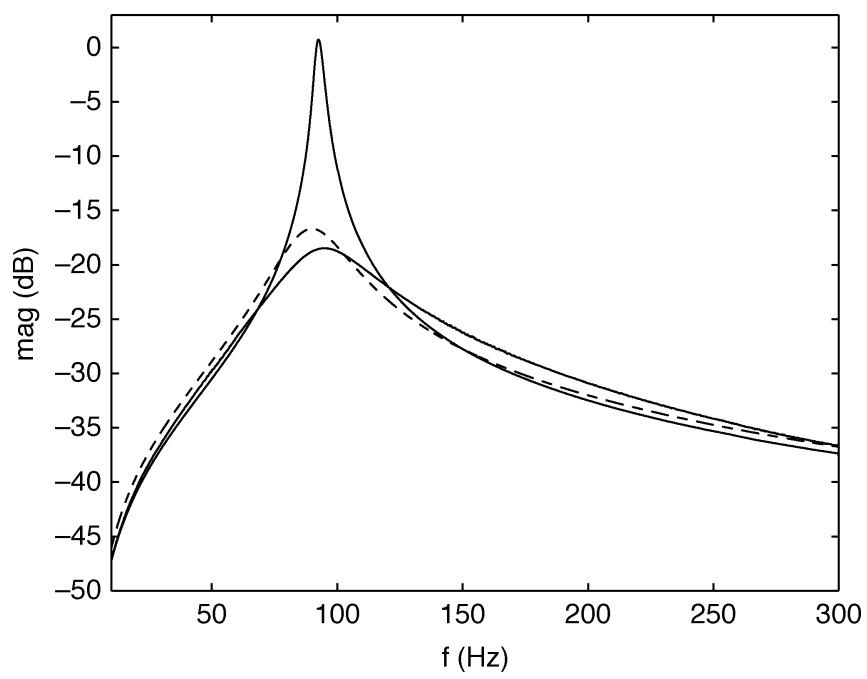

Fig. 26. Experimental (-) and simulated (- -) open- and closed-loop frequency responses from an applied disturbance current $I_{1}$ (A) to the resulting plunger velocity $\nu\left(\mathrm{ms}^{-1}\right)$ for the $\mathcal{H}_{2}$ impedance controlled system. The open-loop frequency response is also shown (-).

and 1, respectively. Our experience indicates that both parameters tend to have a similar effect on the controller bandwidth and closed-loop performance. As either is decreased, the controller bandwidth and closed-loop damping increases.

The electrical shunt impedance of the resulting $\mathcal{H}_{2}$ controller is shown in Fig. 25. Unlike the complex impedance of the LQR controller shown in Fig. 21, the $\mathcal{H}_{2}$ impedance shows a closer resemblance to the ideal negative inductor-resistor over a wide frequency band. Under the same test conditions as discussed in Section IV-C1, the resulting damping performance of the $\mathcal{H}_{2}$ controller is illustrated by the closed-loop frequency response, step response, and pole locations shown in Figs. 26-28, respectively. The damping performance of the $\mathcal{H}_{2}$ controller, measured to be $19.25 \mathrm{~dB}$, is slightly inferior to that obtained for the LQR controller in Section IV-C1. One of the characteristics of the $\mathcal{H}_{2}$ controller was that it resulted in a bandwidth greater than the LQR controller designed to obtain a similar performance. The poor correlation with the simulated response 

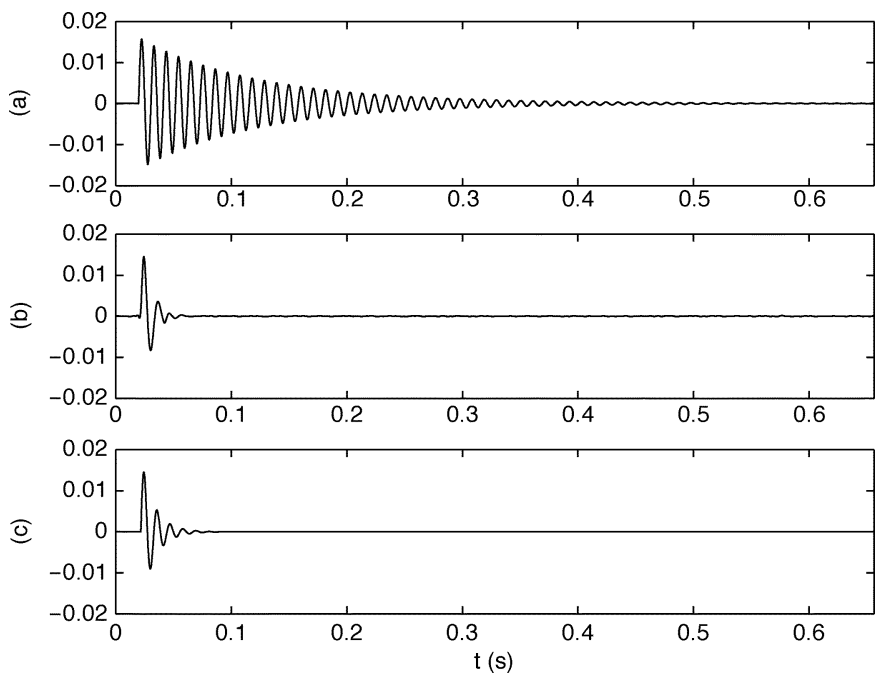

Fig. 27. Velocity response $\nu\left(\mathrm{ms}^{-1}\right)$ of the $\mathcal{H}_{2}$ impedance controlled system to a step disturbance current $I_{1}$. (a) Experimental open loop. (b) Closed loop. (c) Simulated closed loop.

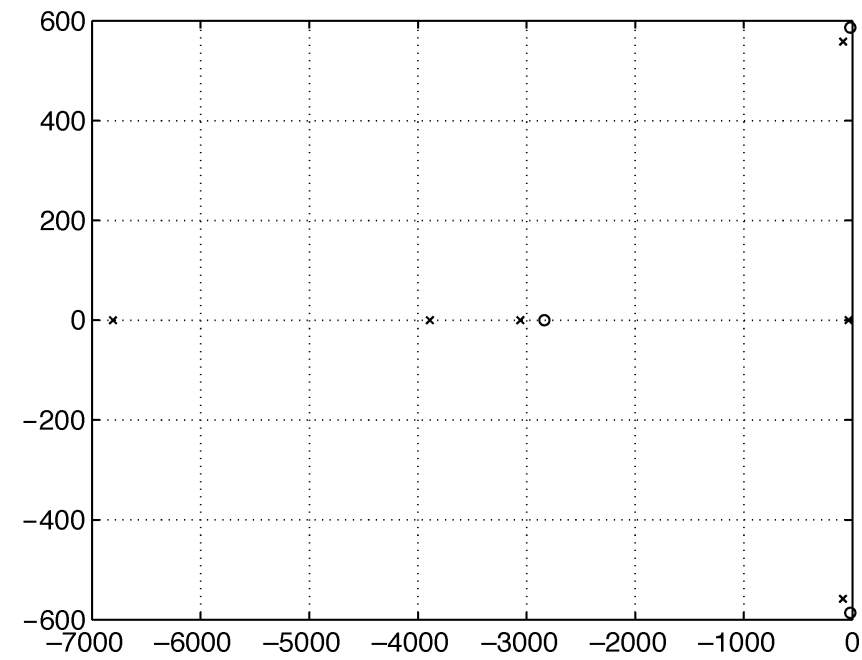

Fig. 28. Open- $(\bigcirc)$ and closed-loop $(x)$ pole locations of the $\mathcal{H}_{2}$ impedance controlled system.

may be attributable to the problems involved in implementing such a high-bandwidth controller. For real-time implementation using the Runge-Kutta analog solver, the sampling delay associated with analog to digital conversion and vice versa results in a significant unmodeled phase delay at frequencies approaching to Nyquist rate. In addition, at such frequencies, the actual impedance presented to the coil is a series concatenation of the implemented transfer function and the high-frequency low-pass dynamics of the power amplifier.

\section{Admittance Synthesis}

Fig. 29 shows the instrumentation and driver gains associated with the underlying electromechanical system. The voltages $V_{1}$ through $V_{4}$ represent the signals applied to, or measured from the power amplifier and instrumentation. The gain and units associated with each signal can be found in Table III. The actual electrical shunt admittance presented to the coil is related to the controller designed for such a system through the gain $k_{4}$ and

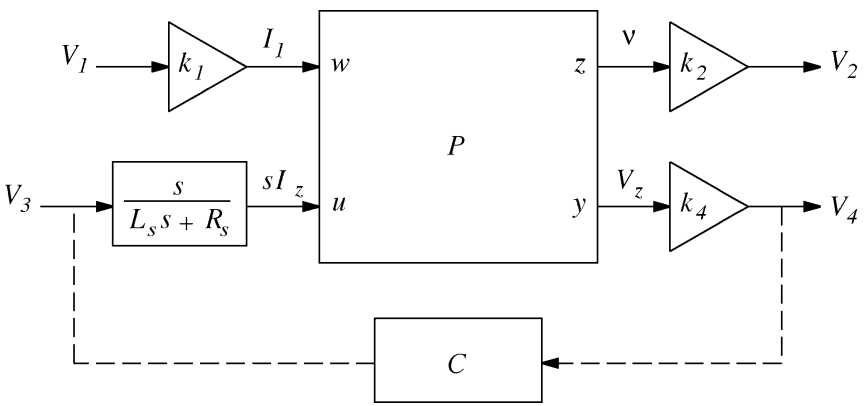

Fig. 29. Open-loop external gains for the shunt current controlled electromagnetic system.

TABLE III

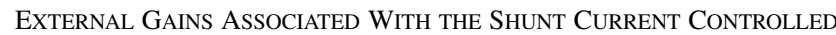
ELECTROMAGNETIC SYSTEM

\begin{tabular}{l|l}
\hline Gain & Value \\
\hline$k_{1}$ & $1 \mathrm{~A} / \mathrm{V}$ \\
\hline$k_{2}$ & $40 \mathrm{~V} / \mathrm{ms}^{-1}$ \\
\hline$L_{s}$ & $466 \mu \mathrm{H}$ \\
\hline$R_{s}$ & $1.1065 \Omega$ \\
\hline$k_{4}$ & $1.77 \mathrm{~V} / \mathrm{V}$ \\
\hline
\end{tabular}
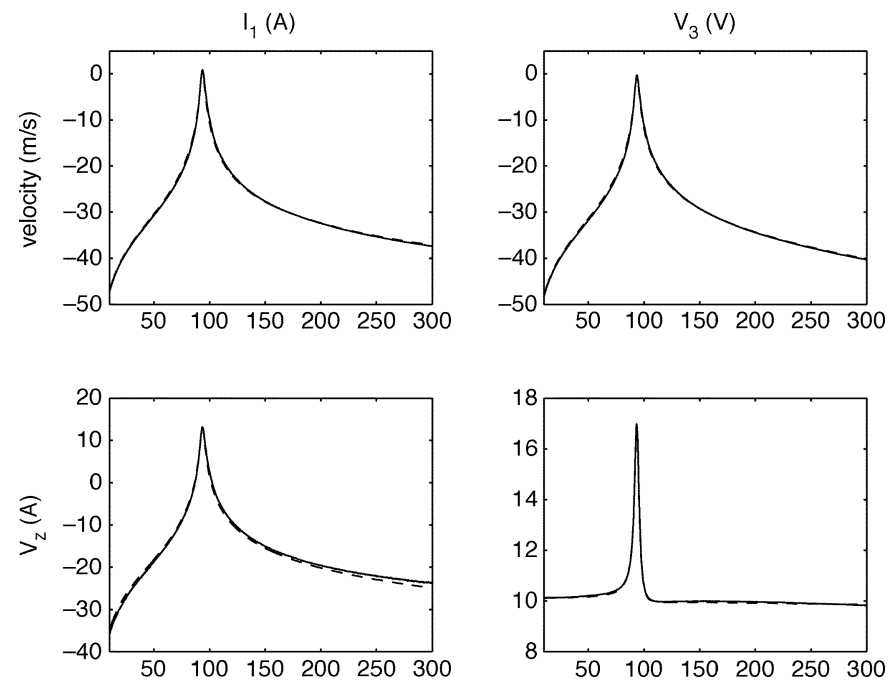

Fig. 30. Simulated (-) and experimental (- -) magnitude frequency response (in decibels) of the shunt current controlled electromagnetic system.

the dynamics of the current fluxion amplifier. It can be shown that

$$
Y_{c}(s)=\frac{I_{z}(s)}{V_{z}(s)}=\frac{1}{L_{s} s+R_{s}} C(s) k_{4} .
$$

The controller $C(s)$ can be likened to the approximation of an admittance derivative, i.e.

$$
C(s) \approx \frac{s L_{s} Y_{c}(s)}{k_{4}} .
$$

In this section, rather than considering only the physical variables such as current, voltage, and velocity etc., we now consider the reference current fluxion $V_{3}(s)$, in preference to $I_{z}(s)$. The magnitude and phase frequency responses are shown, respectively, in Figs. 30 and 31. In the frequency domain, a good 

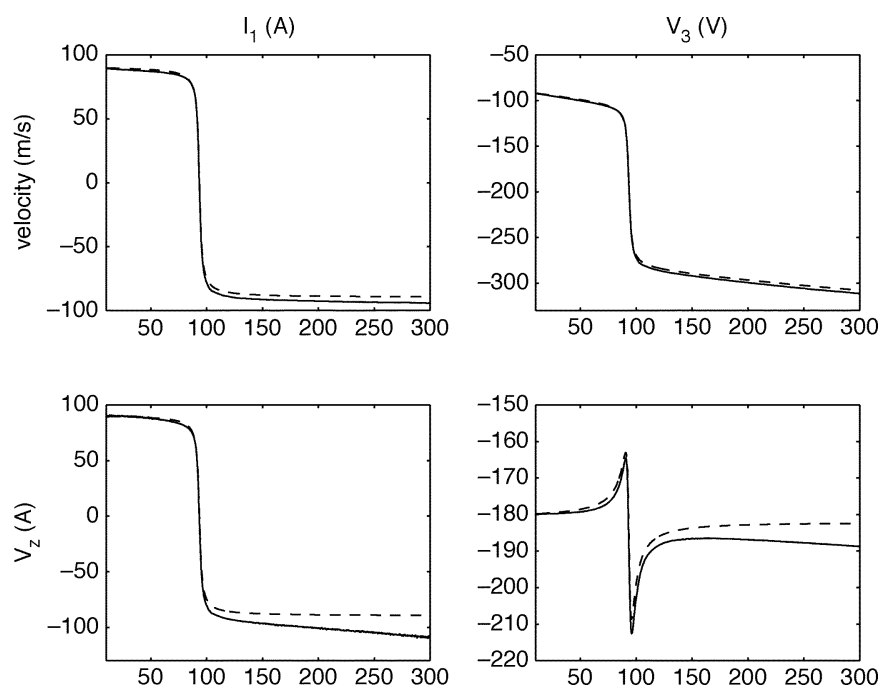

Fig. 31. Simulated (-) and experimental (- -) phase frequency response (in degrees) of the shunt current controlled electromagnetic system.

correlation can be observed between the analytic model and measured system.

1) Admittance Synthesis: In analogy to Section IV-C1, and as discussed in Section III-D, a linear quadratic regulator can be designed to command the shunt current fluxion $V_{3}$ and, hence, the current $I_{z}$. Although $V_{z}$ now appears as an output, it remains in our interest to weight this signal as a component of the performance objective. The connection of the coil and amplifier discussed in Section IV-B has an operational range constrained only by the magnitude of the output voltage.

Based on the physical model (including external gains) that was validated in the previous subsection, an LQR gain matrix was designed to minimize the following performance function:

$$
J=\int_{-\infty}^{\infty}\left[\left(k_{2} \nu(t)\right)^{2}+\left(\frac{k_{y}}{k_{4}} V_{z}(t)\right)^{2}\right] d t
$$

where the factor $k_{y}$ represents the relative weighting on the measured output voltage $V_{z}$. Even though the control and measurement variables differ from the impedance based design in Section IV-C1, the performance objective remains the same. In Section III-D, (45) is cast as a standard LQR performance objective. Following the design of a gain matrix (for $k_{y}=0.5$ ), an observer was designed following the same technique as in Section IV-C1, the poles were chosen with a real component twice that of the closed-loop system poles.

The frequency response of the resulting LQR shunt admittance is shown in Fig. 32. Like the $\mathcal{H}_{2}$ impedance designed and implemented in Section IV-C2, the complex admittance of the LQR controller closely resembles that of the ideal negative inductor-resistor controller at low frequencies. As the frequency increases, the response can be likened to a small and decreasing negative real valued admittance.

The closed-loop response of the LQR controller shown in Figs. 33 and 34 shows a significant peak damping of $20.6 \mathrm{~dB}$, this closely resembles that predicted in simulation. The general coherence between the simulated and experimental results is likely due to the tendency of the LQR admittance to have a significantly lesser bandwidth than that experienced with the
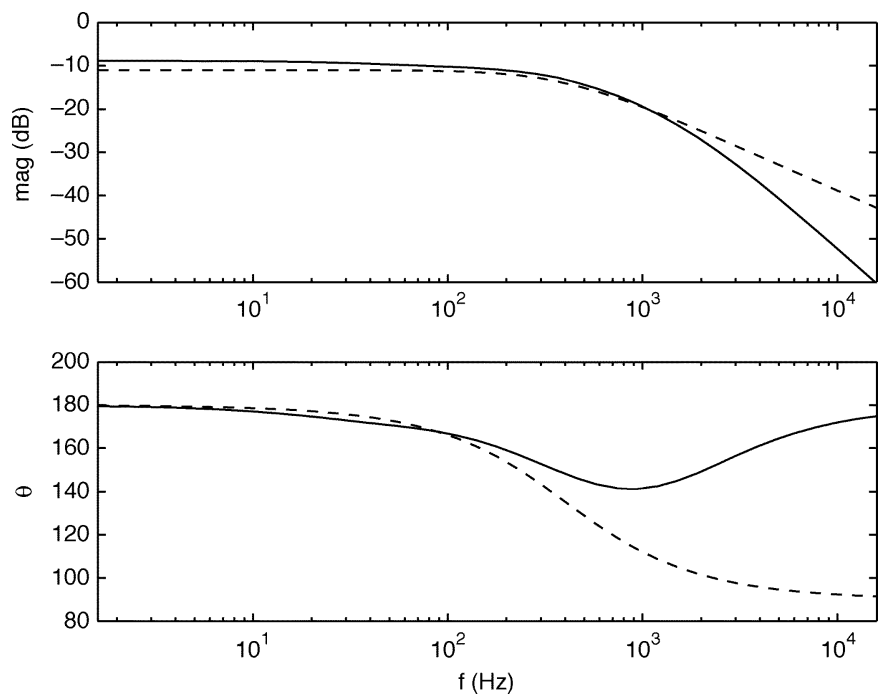

Fig. 32. Complex admittance of the LQR (-) and ideal negative inductor-resistor controller (- -).

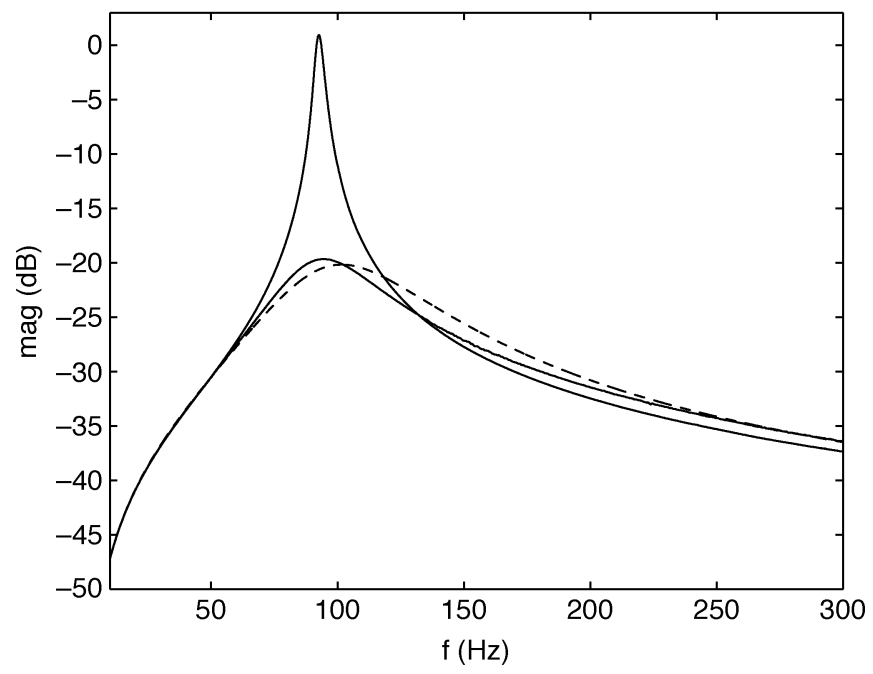

Fig. 33. Experimental (-) and simulated (- -) open- and closed-loop frequency responses from an applied disturbance current $I_{1}$ (A) to the resulting plunger velocity $\nu\left(\mathrm{ms}^{-1}\right)$ for the LQR admittance controlled system. The open-loop frequency response is also shown (-).
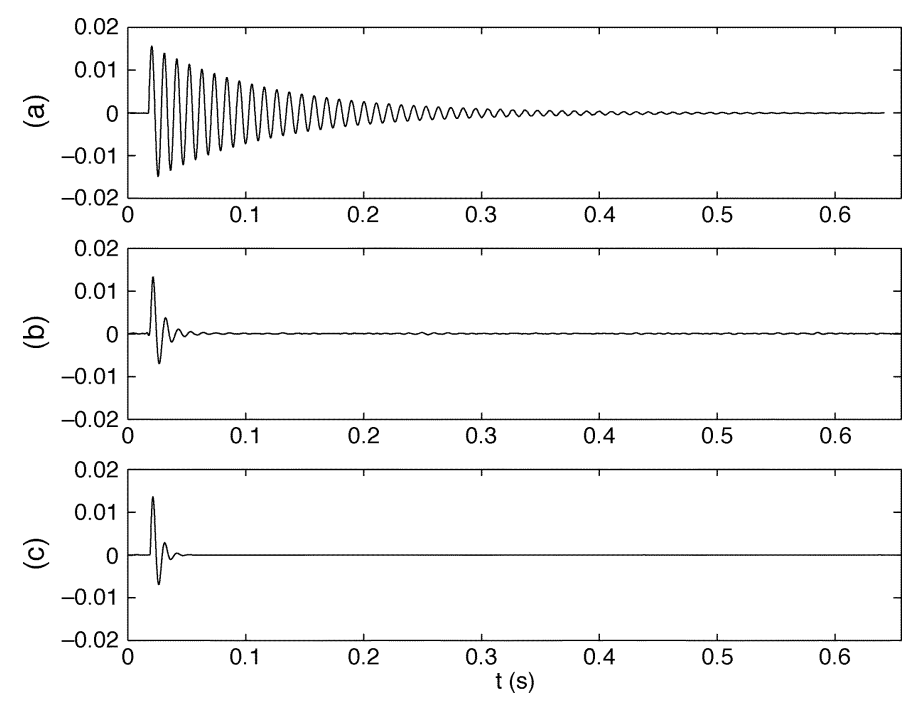

Fig. 34. Velocity response $\nu\left(\mathrm{ms}^{-1}\right)$ of the LQR admittance controlled system to a step disturbance current $I_{1}$. (a) Experimental open loop. (b) Closed loop. (c) Simulated closed loop. 


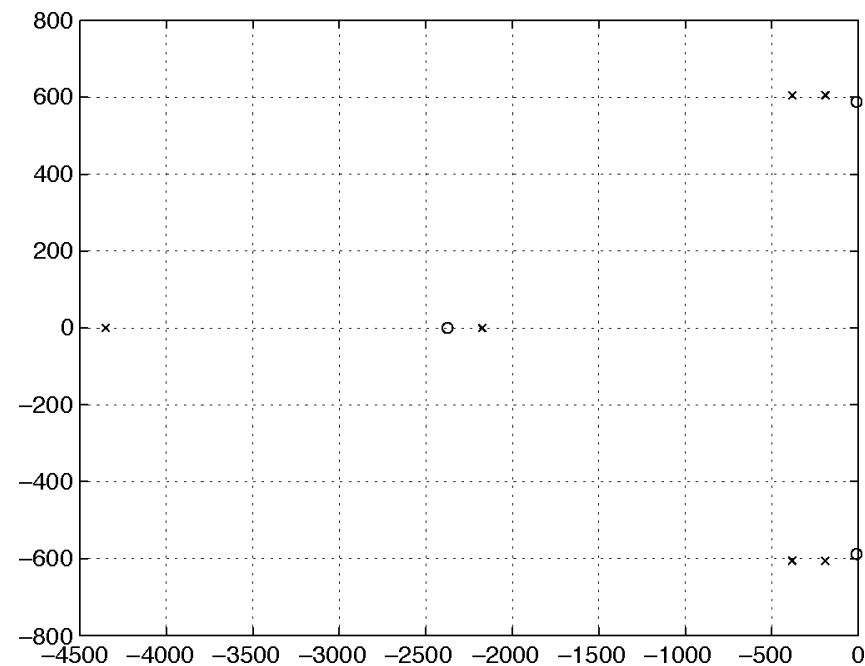

Fig. 35. Open- $(O)$ and closed-loop $(x)$ pole locations of the $L Q R$ admittance controlled system.

impedance controllers of Sections IV-C1 and C-2. As the admittance of the ideal negative inductor-resistor is low-pass compared to the impedance which has an infinite bandwidth, the characteristic of lesser control bandwidth when implementing an admittance follows naturally. In addition, the increase in relative dynamic range associated with the transfer function from $V_{3}$ to $V_{z}$ contributes to a more accurate state estimate and better correlation with simulated results.

The simulated pole locations of the closed-loop system are shown in Fig. 35. As expected, due to the explicit specification of observer poles, the closed-loop pole-zero maps for both the LQR admittance and impedance controlled systems are extremely similar.

2) $\mathcal{H}_{2}$ Admittance Synthesis: This subsection documents the implementation of an active shunt admittance designed to minimize the $\mathcal{H}_{2}$ norm of the transfer function from a disturbance current $I_{1}$ to a performance signal $z$. In analogy to LQR admittance design, the $\mathcal{H}_{2}$ performance function remains unchanged from $\mathcal{H}_{2}$ impedance synthesis. i.e.

$$
J=\left\|\frac{k_{2} \nu(s)+\frac{k_{y}}{k_{3}} V_{z}(s)}{\frac{I_{1}(s)}{k_{1}}}\right\|_{2}
$$

where $k_{y}$ is the weighting on $V_{z}$.

Again, for the plant under consideration, the $\mathcal{H}_{2}$ problem is well defined and feasible. All of the standard requirements are met, i.e., the plant is minimal, proper, controllable, observable, and of finite dimension. However, in order to find a solution using existing tools, i.e., the algebraic Riccati solution implemented by the $\mu$-Synthesis Toolbox for Matlab, the system must meet some additional requirements. The most problematic of these requirements is the requisite full rank condition on the standard plant matrices $D_{21}$ and $D_{12}$. In this case, where each of the signals $w, u, y$, and $z$ are unidimensional, this condition requires that the feedthrough term from $w$ to $y$, and $u$ to $z$, is nonzero. As both of the plant outputs already contain a direct feedthrough from $V_{3}$, (due to the existence of $k_{y}$ ), the only condition not met is that on $D_{21}$. To overcome this problem, for the purpose of controller synthesis, we include an artificial
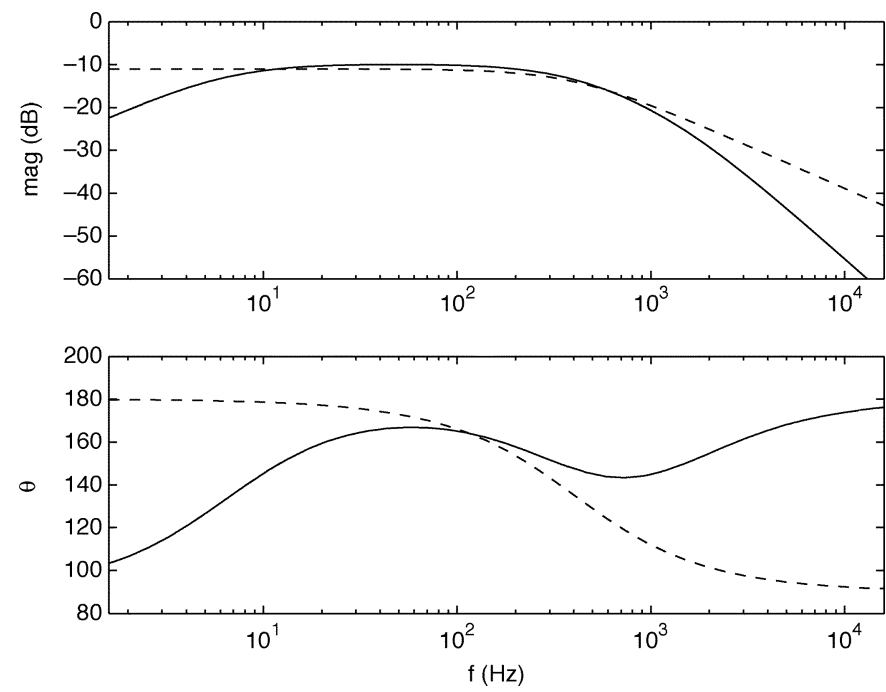

Fig. 36. Complex admittance of the $\mathcal{H}_{2}(-)$ and ideal negative inductor-resistor controller (- -).

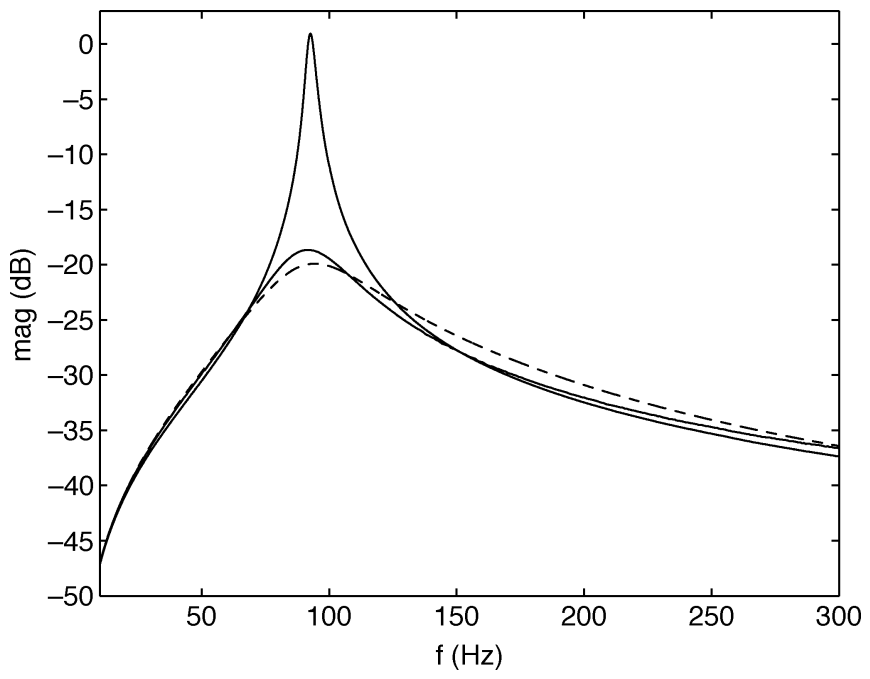

Fig. 37. Experimental (-) and simulated (- -) open- and closed-loop frequency responses from an applied disturbance current $I_{1}$ (A) to the resulting plunger velocity $\nu\left(\mathrm{ms}^{-1}\right)$ for the $\mathcal{H}_{2}$ admittance controlled system. The open-loop frequency response is also shown (-).

feedthrough term $D_{21}$. We now have two design parameters: $k_{y}$ and $D_{21}$. These were chosen to be 0.17 and 0.6 , respectively.

The electrical shunt impedance of the resulting $\mathcal{H}_{2}$ controller is shown in Fig. 36. The damping performance of the $\mathcal{H}_{2}$ controller is assessed from the closed-loop frequency response, step response, and pole locations, as shown in Figs. 35, 37, and 38, respectively. Attenuation of the resonant peak was measured to be $19.65 \mathrm{~dB}$.

\section{E. Discussion}

For the practitioner, when confronted with the various options for shunt control of an electromagnetic system, the obvious questions are: "which is the easiest to implement?" and "which provides the best performance?". Although there is no general answer to either of these questions, we wish to highlight some important characteristics raised throughout this chapter. All of the synthesized LQR and $\mathcal{H}_{2}$ controllers tend to resemble the 

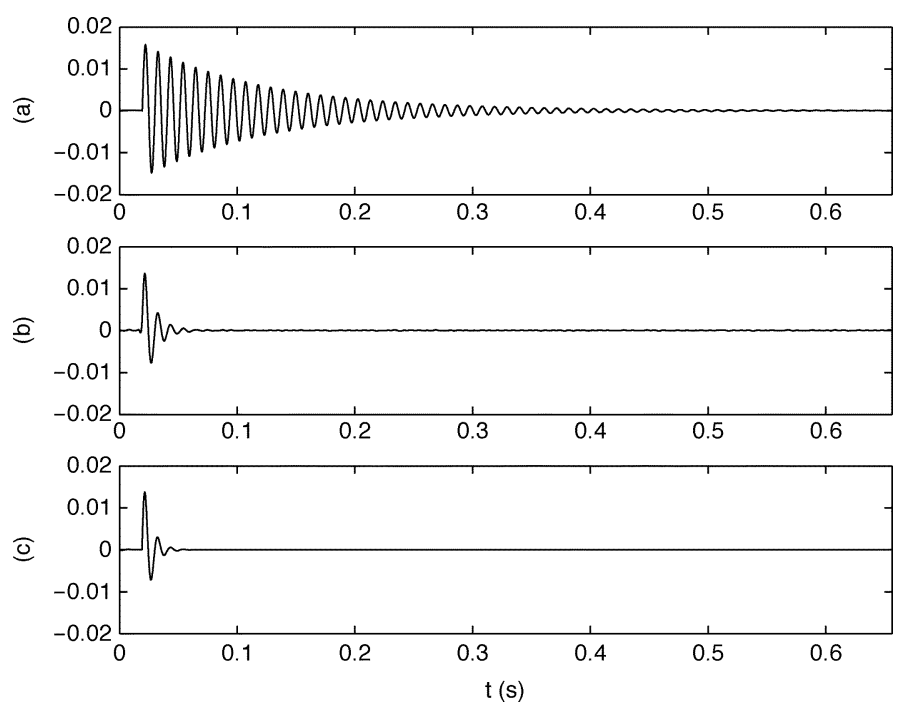

Fig. 38. Velocity response $\nu\left(\mathrm{ms}^{-1}\right)$ of the $\mathcal{H}_{2}$ admittance controlled system to a step disturbance current $I_{1}$. (a) Experimental open loop. (b) Closed loop. (c) Simulated closed loop.

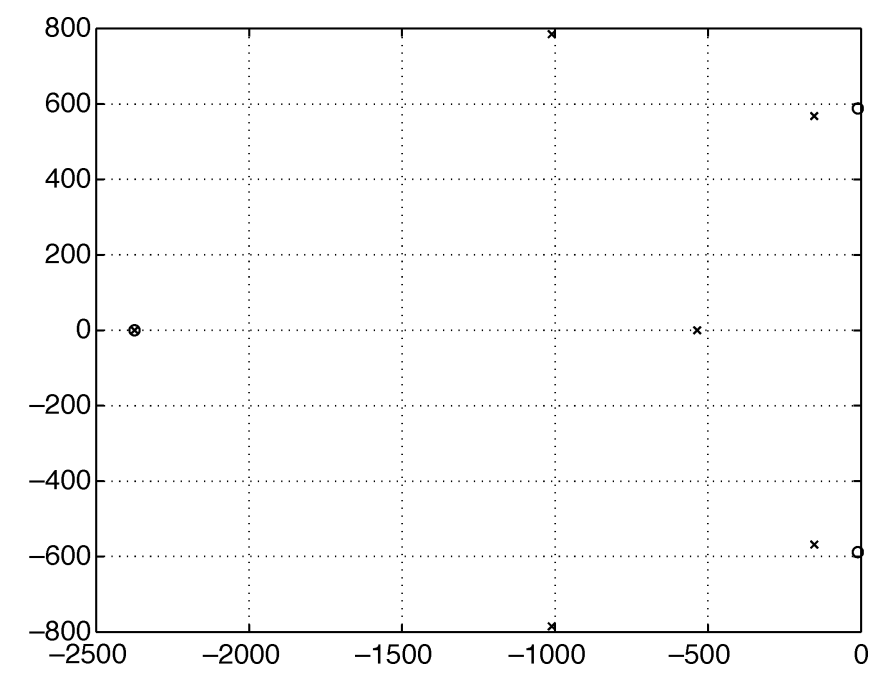

Fig. 39. Open- $(\bigcirc)$ and closed-loop $(\times)$ pole locations of the $\mathcal{H}_{2}$ admittance controlled system.

complex impedance of an ideal negative inductor-resistor over some frequency band. Correspondingly, and as it occurs in practice, the impedance based controllers tend to be of a higher bandwidth and more difficult to implement. Thus, the technique of shunt admittance control is recommended. To this end, a current fluxion amplifier is required. Although such a device is no more difficult to construct than a typical voltage feedback amplifier, at present, they are commercially unavailable.

As there is no direct measurement of the plunger velocity, one would expect the performance of a shunt controlled system to be lesser than a fully instrumented active feedback system. Better performance is typically obtained for systems with a large dynamic range in the transfer function from an applied control signal to the measured variable. Work is continuing on the design of transducers with strong electromagnetic coupling coefficients and, hence, a greater dynamic range in the impedance transfer function.
Another issue associated with electromagnetic shunt control is that of negative reactive components. All of the impedance and admittance transfer functions contained right-half plane poles. Such an impedance can only be represented by a circuit comprising of at least one: negative resistors, negative capacitors, or negative inductors. Although the connection of the coil and control impedance is stable, an inherently stable controller is desirable.

\section{CONCLUSION}

Electromagnetic transducers have been employed extensively in active vibration control systems as force actuators, velocity sensors, or both. Compared to other transducers such as piezoelectric materials and shape memory alloys, their large stroke, physical robustness, high bandwidth, and low-cost render them useful in a wide range of applications.

The connection of an electrical impedance or admittance to the terminals of an electromagnetic coil is equivalent to implementing a standard feedback controller around the mechanical system. By revealing the underlying feedback structure and casting it as a typical MIMO control problem, an impedance or admittance can be found that minimizes some arbitrary performance objective.

No external sensor is required to implement the control loop. This significantly reduces the cost, complexity, and sensitivity to transducer failure that in many applications, may preclude the use of an active control system.

The presented techniques are successfully applied to the design and implementation of an LQR and $\mathcal{H}_{2}$ based, active impedance, and admittance controller. Without the need for any external sensors, the resonant peak of an experimental single DOF system was substantially reduced in magnitude by up to $20.6 \mathrm{~dB}$.

Current and future work involves both the exploration of additional applications and development of the control theory associated with the synthesis step. A priority is the inclusion of uncertainty in the mechanical plant model with consideration during the synthesis process to achieve robust stability and performance objectives. For practical reasons it may also be desirable to enforce strict-positive-realness (passivity) on the synthesis result. It is expected that without negative reactive components the achievable performance will be somewhat lesser. This work has been successfully applied to the control of an isolation support [7], and inertial vibration controller [16].

\section{REFERENCES}

[1] A. Preumont, "Vibration control of active structures," in Solid Mechanics and Its Applications. Norwell, MA: Kluwer, 1997, vol. 50.

[2] S. S. Rao, Mechanical Vibrations, 3rd ed. Reading, MA: AddisonWesley, 1995.

[3] B. M. Hanson, M. D. Brown, and J. Fisher, "Self sensing: Closed-loop estimation for a linear electromagnetic actuator," in Proc. IEEE Amer. Control Conf., Arlington, VA, Jun. 2001, pp. 1650-1655.

[4] C. R. Fuller, S. J. Elliott, and P. A. Nelson, Active Control of Vibration. New York: Academic, 1996.

[5] Y. B. Kim, W. G. Hwang, C. D. Kee, and H. B. Yi, "Active vibration control of suspension system using an electromagnetic damper," in Proc. Int. MECH Eng. Part D: J. Automoblie Engineering, vol. 8, 2001, pp. 865-873.

[6] J. Shaw, "Active vibration isolation by adaptive control," in Proc. IEEE Int. Conf. Control Applications, HI, Aug. 1999, pp. 1509-1514. 
[7] S. Behrens, A. J. Fleming, and S. O. R. Moheimani. Control orientated synthesis of electromagnetic shunt impedances for vibration isolation. presented at Proc. 3rd IFAC Symp. Mechatronic Systems

[8] J. Heng, J. C. Akers, R. Venugopal, M. Lee, A. G. Sparks, P. D. Washabaugh, and D. Bernstien, "Modeling, identification, and feedback control of noise in an acoustic duct," IEEE Trans. Contr. Syst. Technol., vol. 4, no. 3, pp. 283-291, May 1996.

[9] D. Vischer and H. Bleuler, "Self-sensing active magnetic levitation," IEEE Trans. Magn., vol. 29, no. 2, pp. 169-177, Mar. 1993.

[10] C. Choi and K. Park, "Self-sensing magnetic levitation using LC resonant circuits," Sens. Actuators, pp. 1276-1281, 1999.

[11] N. Morse, R. Smith, B. Paden, and J. Antaki, "Position sensed and selfsensing magnetic bearing configuations and associated robustness limitations," in Proc. IEEE Conf. Decision and Control, Tampa, FL, Dec. 1998, pp. 2599-2604.

[12] J. J. Dosch, D. J. Inman, and E. Garcia, "A self-sensing piezoelectric actuator for collocated control," J. Intell. Material Syst. Struct., vol. 3, pp. 166-185, Jan. 1992.

[13] E. H. Anderson, N. W. Hagood, and J. M. Goodliffe, "Self-sensing piezoelectric actuation: Analysis and application to controlled structures," in Proc. AIAA/ASME/ASCE/AHS/ASC Structures, Structural Dynamics, and Materials, 1992, pp. 2141-2155.

[14] S. A. Lane and R. L. Clark, "Improving loudspeaker performance for active noise control," J. Audio Eng. Soc., vol. 46, pp. 508-519, Jun. 1998.

[15] R. L. Clark and K. D. Frampton, "Phase compensation for feedback control of enclosed sound fields," J. Sound Vib., vol. 195, no. 5, pp. 701-718, 1996.

[16] A. J. Fleming, S. Behrens, and S. O. R. Moheimani, "Proof-mass inertial vibration control using a shunted electromagnetic transducer," in Proc. IEEE Conf. Decision and Control, Paradise Island, Bahamas, Dec. 2004, to be published.

[17] S. Behrens, A. Fleming, and S. O. R. Moheimani, "Electrodynamic vibration suppression," in Proc. SPIE Smart Structures and Materials 2003-Damping and Isolation, San Diego, CA, Dec. 2002, Paper 4697-24, pp. 344-355.

[18] C. K. Paulitsch, P. Gardonio, and S. J. Elliott, "Active vibration damping using a self-sensing electrodynamic actuator," in Proc. SPIE Smart Materials and Structures, Damping and Isolation, San Diego, CA, Mar. 2004, pp. 282-293.

[19] S. Behrens, A. J. Fleming, and S. O. R. Moheimani, "A broadband controller for piezoelectric shunt damping of structural vibration," IOP Smart Materials and Structures, vol. 12, pp. 18-28, Jan. 2003.

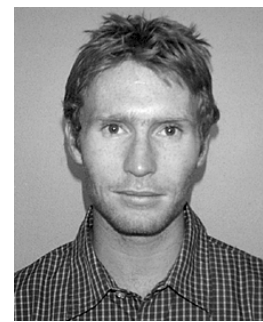

Andrew J. Fleming (M'01) was born in Dingwall, Scotland, in 1977. He received the B.E. (Elec.) and $\mathrm{Ph} . \mathrm{D}$. degrees from The University of Newcastle, Callaghan, Australia, in 2000 and 2004, respectively.

His research involves nanopositioning, and sensorless sound and vibration control.

Dr. Fleming is a member of the Center for Complex Dynamic Systems and Control, an Australian Government Special Research Centre.

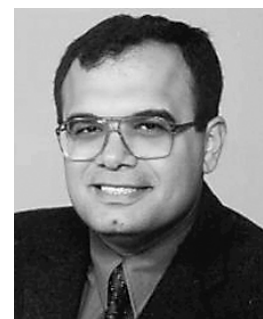

S. O. Reza Moheimani (S'93-M'97-SM'00) received the B.Sc. degree from Shiraz University, Iran, in 1990 and the M.Eng.Sc. and Ph.D. degrees from the University of New South Wales, Australia in 1993 and 1996, respectively, all in electrical and electronics engineering.

In 1996, he was a Postdoctoral Research Fellow at the School of Electrical and Electronics Engineering, Australian Defence Force Academy, Canberra, Australia. In 1997, he joined the University of Newcastle, Australia, where he is currently an Associate Professor in the School of Electrical Engineering and Computer Science. He has over 100 journal and conference publications, is a coauthor of the research monograph Spatial Control of Vibration: Theory and Experiments (Singapore: World Scientific, 2003) and the editor of the volume Perspectives in Robust Control (New York: Springer-Verlag, 2001).

Dr. Moheimani is an Associate Editor for IEEE TRANSACTIONS ON CONTROL Systems TeChNology, Control Engineering Practice, and International Journal of Control, Automation, and Systems. He has served on the editoria boards of several international conferences, and was the Chairman of International Program Committee for the 3rd IFAC Symposium on Mechatronic Systems, held in Sydney, Australia, in 2004. His research interests include smart structures, mechatronic systems, control theory, and signal processing. $\mathrm{He}$ is a Member of the ARC Centre for Complex Dynamic Systems and Control, where he directs the Center's research in the area of mechatronics.

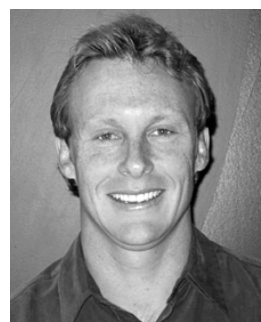

Sam Behrens was born in Sydney, Australia in 1975. He received the B.E. (Mech.) and the M.E. degrees from The University of Newcastle, Australia.

$\mathrm{He}$ is currently a Postdoctoral Research Fellow at the CSIRO Division of Energy Technology, Newcastle, Australia. 